\title{
RESEARCH
}

Open Access

\section{Comparison of the efficacy of hematopoietic stem cell mobilization regimens: a systematic review and network meta-analysis of preclinical studies}

Chengxin Luo ${ }^{1,2+}$, Li Wang ${ }^{3+}$, Guixian Wu ${ }^{1,2}$, Xiangtao Huang ${ }^{1,2}$, Yali Zhang ${ }^{1,2}$, Yanni Ma ${ }^{1,2}$, Mingling Xie ${ }^{1,2}$, Yanni Sun ${ }^{1,2}$, Yarui Huang ${ }^{1,2}$, Zhen Huang ${ }^{1,2}$, Qiuyue Song ${ }^{4}$, Hui Li ${ }^{1,2}, Y u$ Hou ${ }^{1,2}, X i ~ L i^{5^{*}}$, Shuangnian Xu $u^{1,2^{*}}$ and Jieping Chen ${ }^{1,2^{*}}$

\begin{abstract}
Background: Mobilization failure may occur when the conventional hematopoietic stem cells (HSCs) mobilization agent granulocyte colony-stimulating factor (G-CSF) is used alone, new regimens were developed to improve mobilization efficacy. Multiple studies have been performed to investigate the efficacy of these regimens via animal models, but the results are inconsistent. We aim to compare the efficacy of different HSC mobilization regimens and identify new promising regimens with a network meta-analysis of preclinical studies.

Methods: We searched Medline and Embase databases for the eligible animal studies that compared the efficacy of different HSC mobilization regimens. Primary outcome is the number of total colony-forming cells (CFCs) in per milliliter of peripheral blood (/ml PB), and the secondary outcome is the number of $\mathrm{Lin}^{-} \mathrm{Sca}^{+} \mathrm{Kit}^{+}(\mathrm{LSK}) \mathrm{cells} / \mathrm{ml}$ PB. Bayesian network meta-analyses were performed following the guidelines of the National Institute for Health and Care Excellence Decision Support Unit (NICE DSU) with WinBUGS version 1.4.3. G-CSF-based regimens were classified into the SD (standard dose, 200-250 $\mu \mathrm{g} / \mathrm{kg} /$ day) group and the LD (low dose, 100-150 $\mu \mathrm{g} / \mathrm{kg} /$ day) group based on doses, and were classified into the short-term (2-3 days) group and the long-term (4-5 days) group based on administration duration. Long-term SD G-CSF was chosen as the reference treatment. Results are presented as the mean differences (MD) with the associated 95\% credibility interval (95\% Crl) for each regimen.
\end{abstract}

\footnotetext{
* Correspondence: lixi1988xn@163.com; xushuangnian@tmmu.edu.cn; chenjpxn@163.com

${ }^{+}$Chengxin Luo and Li Wang contributed equally to this work.

5 Institute of Infectious Disease, Southwest Hospital, Third Military Medical

University, \#30 Gaotanyan Street, Shapingba District, Chongqing 400038,

China

${ }^{1}$ Center for Hematology, Southwest Hospital, Third Military Medical

University, \#30 Gaotanyan Street, Shapingba District, Chongqing 400038,

China

Full list of author information is available at the end of the article
}

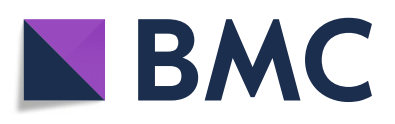

() The Author(s). 2021 Open Access This article is licensed under a Creative Commons Attribution 4.0 International License, which permits use, sharing, adaptation, distribution and reproduction in any medium or format, as long as you give appropriate credit to the original author(s) and the source, provide a link to the Creative Commons licence, and indicate if changes were made. The images or other third party material in this article are included in the article's Creative Commons licence, unless indicated otherwise in a credit line to the material. If material is not included in the article's Creative Commons licence and your intended use is not permitted by statutory regulation or exceeds the permitted use, you will need to obtain permission directly from the copyright holder. To view a copy of this licence, visit http://creativecommons.org/licenses/by/4.0/. The Creative Commons Public Domain Dedication waiver (http://creativecommons.org/publicdomain/zero/1.0/) applies to the data made available in this article, unless otherwise stated in a credit line to the data. 
Results: We included 95 eligible studies and reviewed the efficacy of 94 mobilization agents. Then 21 studies using the poor mobilizer mice model (C57BL/6 mice) to investigate the efficacy of different mobilization regimens were included for network meta-analysis. Network meta-analyses indicated that compared with long-term SD G-CSF alone, 14 regimens including long-term SD G-CSF + Me6, long-term SD G-CSF + AMD3100 + EP80031, long-term SD G-CSF + AMD3100 + FG-4497, long-term SD G-CSF + ML141, long-term SD G-CSF + desipramine, AMD3100 + meloxicam, long-term SD G-CSF + reboxetine, AMD3100 + VPC01091, long-term SD G-CSF + FG-4497, Me6, longterm SD G-CSF + EP80031, POL5551, long-term SD G-CSF + AMD3100, AMD1300 + EP80031 and long-term LD GCSF + meloxicam significantly increased the collections of total CFCs. G-CSF + Me6 ranked first among these regimens in consideration of the number of harvested CFCs/ml PB (MD 2168.0, 95\% Crl 2062.0-2272.0). In addition, 7 regimens including long-term SD G-CSF + AMD3100, AMD3100 + EP80031, long-term SD G-CSF + EP80031, shortterm SD G-CSF + AMD3100 + IL-33, long-term SD G-CSF + ML141, short-term LD G-CSF + ARL67156, and long-term LD G-CSF + meloxicam significantly increased the collections of LSK cells compared with G-CSF alone. Long-term SD G-CSF + AMD3100 ranked first among these regimens in consideration of the number of harvested LSK cells $/ \mathrm{ml}$ PB (MD 2577.0, 95\% Crl 2422.0-2733.0).

Conclusions: Considering the number of CFC and LSK cells in PB as outcomes, G-CSF plus AMD3100, Me6, EP80031, ML141, FG-4497, IL-33, ARL67156, meloxicam, desipramine, and reboxetine are all promising mobilizing regimens for future investigation.

Keywords: Hematopoietic stem cells, Mobilization, Animal studies, Network meta-analysis

\section{Background}

Hematopoietic stem cell transplantation (HSCT) is a lifesaving strategy for variety of severe disorders, including bone marrow failure after high-dose radiation and various hematological malignancies [1]. Peripheral blood stem cells (PBSCs) have been gradually replaced bone marrow (BM) as the predominant source of stem cell for transplantation in clinical practice [2,3]. It has been demonstrated that PBSCs transplantation is associated with more convenient and safer harvest procedure, faster hematological recovery, lower risk of graft failure, and comparable disease-free survival (DFS) and overall survival (OS) in comparison with BM transplantation [4-6]. However, hematopoietic stem cells (HSCs) mainly reside in specialized BM niches during steady state; the number of HSCs circulating in peripheral blood (PB) is very low and not sufficient for harvest [6]. Administration of exogenous cytokines or chemokines could induce the egress of HSCs from BM into PB in a process termed mobilization. Successful mobilization allows for efficient collection of HSCs sufficient for transplantation, and increment in the dose of harvested HSCs could improve transplantation efficiency via promoting hematopoietic reconstitution, as well as reducing the need for supportive blood transfusion and the risks of infections [7, 8]. Therefore, efficient mobilization is the key to successful HSCT and sustained hematopoietic recovery.

Granulocyte colony-stimulating factor (G-CSF) is the most commonly used steady-state HSC mobilization agent in clinical practice. However, mobilization failure may occur when G-CSF is used alone [8]. In addition, mobilization using G-CSF alone requires multiple doses beginning at least 4 days before first apheresis and a median of 2-5 apheresis sessions to collect sufficient PBSCs, which increased the risk of adverse events [7]. The incidences of bone pain induced by G-CSF is higher than $80 \%$ at day 4 , in addition, other G-CSF-related severe adverse events including myocardial infarctions, pulmonary embolism, and splenic rupture also have been reported [9-11]. To improve mobilization efficacy and attenuate toxicity, novel mobilization regimens are developed and investigated in a variety of animal studies before applied in clinical practice, but the results are inconsistent. This study aims to review and compare the efficacy of different HSC mobilization regimens and identify new promising regimens with a network metaanalysis of preclinical studies, which may be helpful for guiding future clinical trials.

\section{Methods}

\section{Literature search and study selection}

We searched Medline and Embase from inception to February 23, 2021, with the search term "stem cell mobilization" and a filter of "animals". The titles and abstracts of retrieved citations were independently screened by two investigators (CXL and XL) for eligibility. Disagreements were resolved by full-text review and discussion with a third investigator (SNX). Preclinical studies that met the following criteria were included for review: (1) compared the efficacy of two or more different regimens in the mobilization of hematopoietic stem and progenitor cells (HSPCs) and (2) using any species of mice as experimental animals. As for network metaanalysis, the inclusion criteria were (1) using the poor mobilizer mice model-C57BL/6 mice as experimental 
animals [12] and (2) reporting data for at least one of the outcomes of mobilization efficacy, including the number of total colony-forming cells (CFCs) and $\mathrm{Lin}^{-}$ $\mathrm{Sca}^{+} \mathrm{Kit}^{+}$(LSK) cells per milliliter of peripheral blood (/ml PB). Since aged mice were reported to have better mobilization efficiency compared with young mice and no significant difference was reported among mice younger than 3 months, we excluded studies using mice older than 12 weeks in meta-analysis to reduce heterogeneity [13]. In addition, we only included studies that administrated G-CSF via subcutaneously injection. Furthermore, G-CSF-based regimens were classified into the SD (standard dose, 200-250 $\mu \mathrm{g} / \mathrm{kg} /$ day) group and the LD (low dose, $100-150 \mu \mathrm{g} / \mathrm{kg} /$ day) group based on G-CSF doses and were classified into the short-term (2-3 days) group and the long-term (4-5 days) group based on administration duration of G-CSF. Studies with significant heterogeneity in dosage and injection route of G-CSF were excluded in meta-analysis.

\section{Data extraction and quality assessment}

Full text of all eligible studies was reviewed, and two investigators (CXL and $\mathrm{XL}$ ) independently extracted data using predesigned data collection forms. Data was extracted on studies characteristics, animal's characteristics, dosage of mobilization regimens, and efficacy outcomes. We chose the number of total CFCs per milliliter PB as primary outcome, and the number of LSK cells per milliliter PB as secondary outcome. The mean, standard deviation (SD) or standard error (SE) of each outcome are extracted directly from published text or from related graphs with Adobe Photoshop version CS3 via previously validated methods [14]. The methodological quality of included studies was assessed using the SYstematic Review Centre for Laboratory animal Experimentation (SYRCLE) risk of bias tool, which contains 10 items, including random sequence generation, similar baseline characteristics, allocation concealment, random housing, blinding of caregivers and investigators, random selection for outcome assessment, blinding of outcome assessor, adequate addressing of incomplete outcome data, free from selective outcome reporting, and free from other bias [15]. For each item, judgment of "yes", "no", and "unclear" respectively indicate low, high and unclear risk of bias.

\section{Statistical analyses}

We conducted network meta-analyses to compare the efficacy of multiple mobilization regimens simultaneously. Network plot for each outcome was obtained using Stata version 12.0. Bayesian network meta-analyses were performed with WinBUGS version 1.4.3 (MRC Biostatistics Unit, Cambridge, UK), employing the Markov Chain Monte Carlo (MCMC) approach and following the guidelines of the National Institute for Health and Care Excellence Decision Support Unit (NICE DSU) [16]. We used the WinBUGS code previously established by Dias et al., which could handle trials with multiple arms and rank treatments with additional code [16]. Three chains were run to yield 150,000 iterations, and the initial 5000 burn-ins were discarded. The convergence of models was assessed with trace plots and Brooks-Gelman-Rubin statistic. Model fit of fixed-effect model and random-effect model were compared with the Deviance Information Criterion (DIC), and model with lower DIC was adopted. Long-term SD G-CSF monotherapy was chosen as the common comparator. Estimates of treatment effects were reported as mean differences (MD) with the associated 95\% credibility interval (95\% CrI). The 95\% CrI calculated in Bayesian meta-analysis can be interpreted like the $95 \%$ confidence intervals $(95 \% \mathrm{CI})$ in traditional meta-analysis [17]. The probability of each regimen to be the best was calculated by ranking the relative effects of all treatments in each iteration and defined as the proportion of times a regimen ranked first. This work is reported according to the Preferred Reporting Items for Systematic Reviews and Meta-analyses (PRISMA) extension statement for network meta-analyses [18].

\section{Results}

\section{Characteristics of included trials}

We identified 3826 records from database searches. After removing 835 duplicates, 2991 records were screened on title and abstract, and 2749 clearly irrelevant records were excluded. We retrieved the full text of the remaining 242 records for further assessment. We excluded 147 records for the reasons listed in the flow diagram (Fig. 1). Ultimately, 95 eligible studies were included for review [19-113]. The characteristics of the 95 included studies are summarized in Supplementary Table 1. The efficacy of 94 HSC mobilization agents were investigated, including cytokines, agents targeting the CXCR4 (C-X-C chemokine receptor type 4)/ CXCL12 (SDF1, stromal cell-derived factor-1) axis, agents targeting the VLA-4(very late antigen-4)/VCAM1 (vascular cell adhesion molecule-1) axis, chemotherapeutic agents, nonsteroidal anti-inflammatory drugs (NSAIDs), and other agents. Most of these agents not only can induce the mobilization of hematopoietic stem and progenitor cells (HSPCs) alone, but also can enhanced the mobilization mediated by G-CSF or AMD3100 synergistically or additively. The detailed information and mobilization efficacy of these agents are reviewed in Table 1. Compared with the conventional G-CSF, modified G-CSF including SD/0 (an engineered pegylated G-CSF), IMG-CSF (G-CSF immobilized on polyethylenoxide by nanotechnology), and PEGLip-G- 


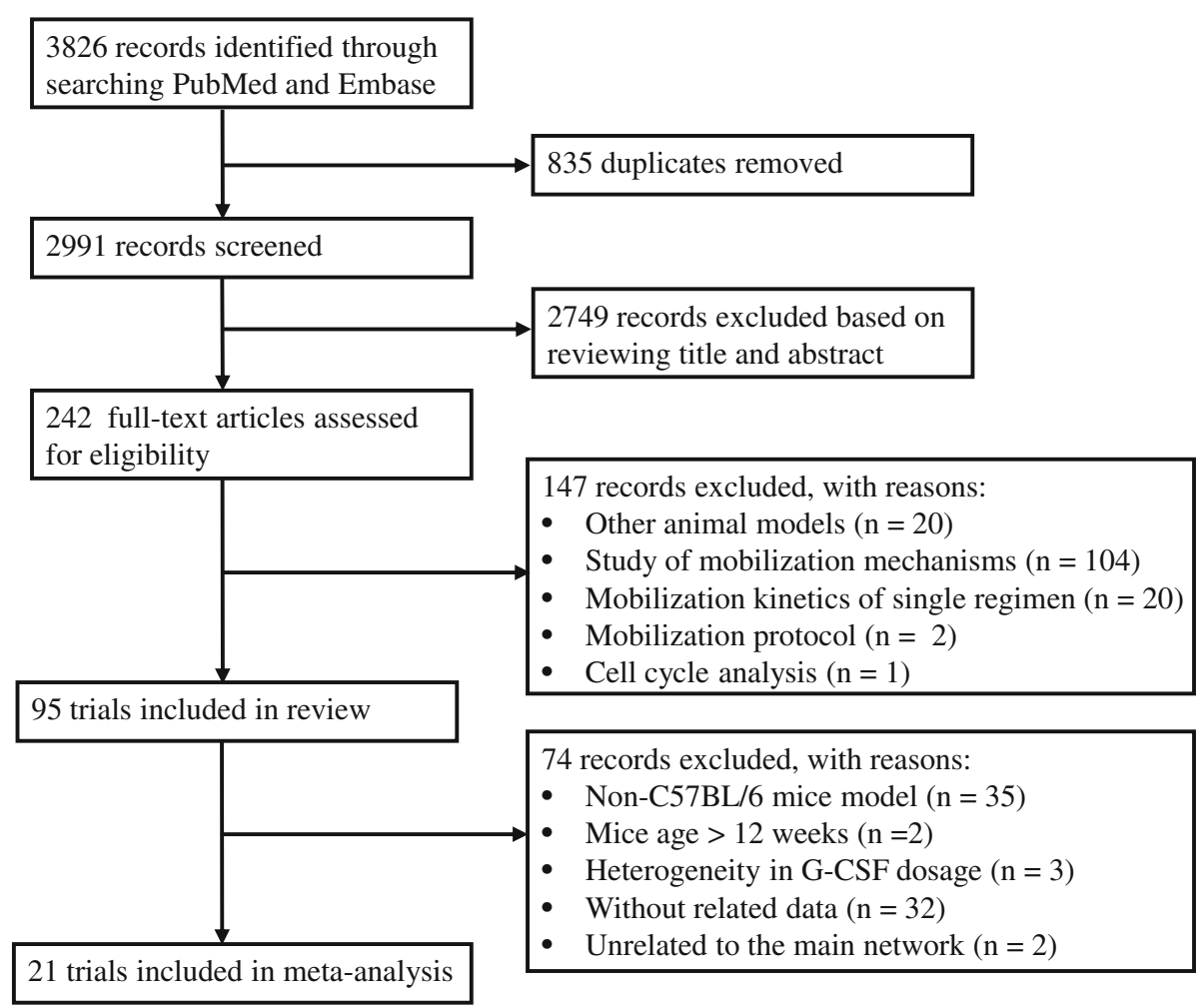

Fig. 1 Flow chart of study selection. The PRISMA flow chart of study screening and selection

CSF (pegylated liposome formulated G-CSF) exhibited enhanced mobilization efficacy. Among other cytokines, IL-33 showed superior mobilization potential than GCSF and AMD3100, and tGRO $\beta$ (a truncated form of chemokine GRO $\beta$ ) showed superior mobilization AMD3100. AMD3100-a CXCR4 antagonist was the most commonly used agents in combined regimens, which can significantly increase mobilization with a single dose when in combination with G-CSF. There are 7 new CXCR4 antagonist investigated and compared with AMD 3100, among which T-140, POL5551, and CX0714 showed significant superior mobilization AMD3100.

After excluding studies using non-C57BL/6 mice model, studies including mice older than 12 weeks, studies that did not reported data about the number of total CFCs or LSK cell per milliliter PB, studies with significant heterogeneity in G-CSF dosage, and studies that were unrelated to the main network, 21 eligible studies were included in meta-analysis $[51,56,72,74,77,78$, $81-86,91,94,96,104-106,108,109,112]$. All of the 21 included studies are controlled studies, and the most widely used controls are phosphate-buffered saline (PBS), saline, and G-CSF. There are 40 mobilization agents and 57 regimens investigated. The characteristics of these 21 studies are summarized in Table 2 . The results of methodological quality evaluation are listed in Supplementary Table 2. Risk of bias regarding random allocation and blinding in all included studies are unclear since the lack of relevant information. The baseline characteristics including mice strain and gender are unified among groups in 10 studies, the other 11 studies did not report the animal gender and age. There are 2 studies that only reported representative data for mobilization outcomes; the other studies are all free from bias caused by incomplete outcome data, selective outcome reporting, and other reasons.

\section{Total CFCs}

The number of total CFCs (also known as colonyforming units, CFUs) per milliliter of PB was reported as primary outcome in 17 studies and involved 43 mobilization regimens. The network graph of all comparisons in these 17 studies is shown in Fig. 2. The results of Bayesian network meta-analysis indicate that compared with long-term SD G-CSF alone, 14 mobilization regimens significantly increased the number of total CFCs $/ \mathrm{ml} \mathrm{PB}$, including long-term SD G-CSF + Me6 (MD 2168.0, 95\% CrI 2062.0-2272.0), long-term SD G-CSF + AMD3100 + EP80031 (MD 1144.0, 95\% CrI 974.9-1311.0), long-term SD G-CSF + AMD3100 + FG-4497 (MD 903.9, 95\% CrI 727.5, 1080.0), long-term SD G-CSF + ML141 (MD 720.9, 95\% CrI 567.1-875.3), long-term SD G-CSF + desipramine (MD 594.7, 95\% CrI 419.4-768.8), AMD3100 + meloxicam (MD 580.1, 95\% 
Table 1 Detailed information and mobilization efficacy of all included novel agents

\begin{tabular}{|c|c|c|c|}
\hline Agent & Detailed information & HSPC mobilization efficacy & References \\
\hline \multicolumn{4}{|l|}{ 1. Cytokines } \\
\hline SCF & Stem cell factor & $\begin{array}{l}\text { Multi-day SCF alone can induce mobilization; IL- } \\
11 \text { plus SCF synergistically enhanced } \\
\text { mobilization. }\end{array}$ & Mauch et al. [21] \\
\hline $\mid \mathrm{L}-11$ & Interleukin-11 & $\begin{array}{l}\text { Multi-day IL-11 alone can induce mobilization; IL- } \\
11 \text { plus SCF or G-CSF synergistically enhanced } \\
\text { mobilization. }\end{array}$ & Mauch et al. [21], Meng et al. [42] \\
\hline MIP-1a & $\begin{array}{l}\text { Macrophage inflammatory protein } \\
\text { 1a }\end{array}$ & $\begin{array}{l}\text { Single-dose MIP-1a alone induced rapid } \\
\text { mobilization; MIP-1a markedly enhanced G-CSF- } \\
\text { and AMD3100-induced mobilization. }\end{array}$ & Lord et al. [20], Broxmeyer et al. [52] \\
\hline IL-8 & Interleukin-8 & $\begin{array}{l}\text { Single-dose IL-8 alone induced rapid } \\
\text { mobilization; the combination of IL- } 8 \text { with G-CSF } \\
\text { or CWHM-823 enhanced mobilization. }\end{array}$ & $\begin{array}{l}\text { Wang et al. [24], Zhang et al. [28], de } \\
\text { Kruiif et al. [66], Karpova et al. [107] }\end{array}$ \\
\hline MIP-2 & $\begin{array}{l}\text { Macrophage inflammatory protein- } \\
2\end{array}$ & $\begin{array}{l}\text { Single-dose MIP-2 alone induced rapid } \\
\text { mobilization; MIP-2 markedly enhanced G-CSF- } \\
\text { induced mobilization. }\end{array}$ & Wang et al. [24] \\
\hline FLT-3L & Fms-like tyrosine kinase-3 ligand & $\begin{array}{l}\text { Multi-dose FLT-3L alone can induce mobilization; } \\
\text { FLT-3L synergistically enhanced mobilization in- } \\
\text { duced by G-CSF, GM-CSF, IL-8, and AMD } 3100 \text {. }\end{array}$ & $\begin{array}{l}\text { Brasel et al. [22], Sudo et al. [23], Neipp } \\
\text { et al. [25], Robinson et al. [33], Robinson } \\
\text { et al. [44], de Kruijf et al. [66], He et al. } \\
\text { [83] }\end{array}$ \\
\hline GM-CSF & $\begin{array}{l}\text { Granulocyte macrophage colony- } \\
\text { stimulating factor }\end{array}$ & $\begin{array}{l}\text { GM-CSF alone did not induce significant } \\
\text { mobilization; FLT-3L + GM-CSF synergistically en- } \\
\text { hanced mobilization. }\end{array}$ & Brasel et al. [22]; Robinson et al. [33] \\
\hline PEG-MGDF & $\begin{array}{l}\text { Pegylated megakaryocyte growth } \\
\text { and development factor }\end{array}$ & $\begin{array}{l}\text { Multi-day PEG-MGDF alone can induce } \\
\text { mobilization; PEG-MGDF synergizes with G-CSF } \\
\text { to enhance mobilization. }\end{array}$ & Torii et al. [27], Honda et al. [36] \\
\hline $\begin{array}{l}\mathrm{SD} / 01^{\mathrm{a}}, \mathrm{IM} \mathrm{G}- \\
\mathrm{CSF}^{\mathrm{a}} \text {, and } \\
\text { PEGLip-G-CSF }\end{array}$ & Modified G-CSF & $\begin{array}{l}\text { Modified G-CSF exhibited superior mobilization } \\
\text { potential compared with standard G-CSF }\end{array}$ & $\begin{array}{l}\text { De Haan et al. [30], Dygai et al. [62], } \\
\text { Yatuv et al. [65] }\end{array}$ \\
\hline $\begin{array}{l}\text { GRO } \beta \text { and } \\
\mathrm{tGRO} \beta^{\mathrm{b}}\end{array}$ & $\begin{array}{l}\text { GRO } \beta \text { : CXCL2, a chemokine; } \mathrm{tGRO} \beta \\
\text { truncated form of } \mathrm{GRO} \beta \text { (also } \\
\text { known as SB-251353, GRO } \beta_{\mathrm{T}} \\
\text { GRO } \beta_{\triangle 4} \text { ) }\end{array}$ & $\begin{array}{l}\text { Single-dose GRO } \beta \text { or } t G R O \beta \text { alone induced rapid } \\
\text { mobilization; tGRO- } \beta \text { synergistically enhanced } \\
\text { the mobilization effects of G-CSF, AMD3100, and } \\
\text { VLA-4 antagonists. }\end{array}$ & $\begin{array}{l}\text { King et al. [37], Pelus et al. [47], Fukuda } \\
\text { et al et al. [54], Hoggatt et al. [77], } \\
\text { Karpova et al. [107] }\end{array}$ \\
\hline rhPIGF-1 & $\begin{array}{l}\text { Recombinant human placental } \\
\text { growth factor-1 }\end{array}$ & $\begin{array}{l}\text { rhPIGF-1 alone had no mobilizing activity; } \\
\text { rhPIGF-1 synergized with G-CSF in mobilization. }\end{array}$ & Carlo-Stella et al. [53] \\
\hline LECT2 & $\begin{array}{l}\text { Leukocyte cell-derived chemotaxin } \\
2\end{array}$ & $\begin{array}{l}\text { 5-day LECT2 alone induced mobilization; the } \\
\text { combination of LECT2 synergistically enhanced } \\
\text { AMD3100 - but not G-CSF-induced } \\
\text { mobilization. }\end{array}$ & Lu et al. [91] \\
\hline GROa & The CXCR2 ligand CXCL1 & $\begin{array}{l}\text { Single-dose GROa alone induced rapid } \\
\text { mobilization, the combination of GROa with } \\
\text { CWHM- } 823 \text { enhanced mobilization. }\end{array}$ & Karpova et al. [107] \\
\hline $1 \mathrm{LL}-33^{\mathrm{ab}}$ & Interleukin-33 & $\begin{array}{l}\text { 3-day IL-33 alone mobilized HSPCS more effi- } \\
\text { ciently than G-CSF or AMD3100; IL-33 additively } \\
\text { enhanced G-CSF- and AMD 3100-induced } \\
\text { mobilization. }\end{array}$ & Alt et al. [105] \\
\hline CSF1-FC & CSF1 Fc fusion protein & CSF1-Fc enhanced G-CSF-induced mobilization. & Kaur et al. [113] \\
\hline
\end{tabular}

\section{Agents targeting CXCR4/CXCL12 (SDF1) axis}

2.1 CXCR4 antagonists

$\begin{array}{ll}\text { AMD3100 Plerixafor, a CXCR4 antagonist } \\ \text { T-140 } & \begin{array}{l}\text { 4F-benzoyl-TN14003, a highly } \\ \text { selective CXCR4 antagonist }\end{array}\end{array}$

TG-0054 A novel CXCR4 antagonist

\section{1 plus SCF synergistically enhanced}

Multi-day IL-11 alone can induce mobilization; IL- Mauch et al. [21], Meng et al. [42]

11 plus SCF or G-CSF synergistically enhanced

zation. mobilization; MIP-1a markedly enhanced G-CSFand AMD3100-induced mobilization. mobilization; the combination of IL-8 with G-CSF

mobilization; MIP-2 markedly enhanced G-CSFinduced mobilization.

FLT-3L synergistically enhanced mobilization induced by G-CSF, GM-CSF, IL-8, and AMD 3100. hanced mobilization to enhance mobilization

Modified G-CSF exhibited superior mobilization potential compared with standard G-CSF

Single-dose GRO $\beta$ or $t G R O \beta$ alone induced rapid mobilization; tGRO- $\beta$ synergistically enhanced VLA-4 antagonists.

rhPIGF-1 alone had no mobilizing activity; synergized with G-CSF in mobilization. combintion of LECT2 synergistically enhanced AMD3100 - but not G-CSF-induced mobilization.

3-day IL-33 alone mobilized HSPCs more effienhanced G-CSF- and AMD 3100-induced

CSF1-Fc enhanced G-CSF-induced mobilization.

Kaur et al. [113]
Single-dose AMD 3100 alone can induce rapid mobilization; AMD 3100 synergizes with G-CSF to mobilize HSPCs.

T-140 has superior mobilization potential than AMD 3100; T-140 synergizes with G-CSF to mobilize HSPCs with higher efficacy than G-CSF + AMD 3100.

Single-dose TG-0054 alone can induce
Broxmeyer et al. [48]; Abraham et al. [51], Bonig et al. [61]

Abraham et al. [51]

Huang et al. [63] 
Table 1 Detailed information and mobilization efficacy of all included novel agents (Continued)

\begin{tabular}{|c|c|c|}
\hline Agent & Detailed information & HSPC mobilization efficacy \\
\hline & & $\begin{array}{l}\text { mobilization; TG-0054 showed synergistic effects } \\
\text { when combined with G-CSF. }\end{array}$ \\
\hline POL5551 & A novel peptidic CXCR4 antagonist & $\begin{array}{l}\text { Single-dose POL5551 induced higher levels of } \\
\text { mobilization than AMD 3100; POL5551 synergizes } \\
\text { with G-CSF and CY in mobilization; continuous } \\
\text { infusion of POL5551 for 1-2 weeks achieved } \\
\text { higher mobilization than G-CSF. }\end{array}$ \\
\hline ALT1188 & A small molecule CXCR4 antagonist & $\begin{array}{l}\text { Single-dose ALT1188 alone can induce rapid } \\
\text { mobilization; Continuous infusion of ALT1188 for } \\
2 \text { weeks achieved higher mobilization than G- } \\
\text { CSF. }\end{array}$ \\
\hline KRH3955 & $\begin{array}{l}\text { A chemically distinct CXCR4 } \\
\text { antagonist }\end{array}$ & $\begin{array}{l}\text { Single-dose KRH3955 alone induced rapid } \\
\text { mobilization; the combination of KRH3955 with } \\
\text { AMD } 3100 \text { did not enhance mobilization. }\end{array}$ \\
\hline$C \times 0714^{b}$ & $\begin{array}{l}\text { A selective and potent CXCR4 } \\
\text { antagonist }\end{array}$ & $\begin{array}{l}\text { CX0714 has greater mobilization ability than } \\
\text { AMD 3100; CX0714 synergistically enhanced G- } \\
\text { CSF-induced mobilization with higher efficacy } \\
\text { than G-CSF + AMD3100. }\end{array}$ \\
\hline HF51116 & $\begin{array}{l}\text { A small molecule antagonist of } \\
\text { CXCR4 }\end{array}$ & $\begin{array}{l}\text { The mobilization efficacy of HF51116 was } \\
\text { comparable to AMD 3100; HF51116 } \\
\text { synergistically enhanced G-CSF-induced } \\
\text { mobilization. }\end{array}$ \\
\hline
\end{tabular}

\section{References}

Karpova et al. [78], Karpova et al. [94]

Karpova et al. [94]

Redpath et al. [97]

Wu et al. [103]

Fang et al. [112]

\subsection{Other agents targeting CXCR4/CXCL12 axis}

\section{CTCE-0021 \\ An SDF-1 analog}

SCA

Sulfated colominic acid, a compound that can modulate CXCR4 function

ATI-2341 A pepducin CXCR4 agonist

APACs (Neo- Compounds that can compete r9, Neam-r9) with CXCL12 binding to CXCR4 and $r 9$

NOX-A 12

A mirror-image oligonucleotide inhibitor of CXCL12

$\mathrm{Me6}^{\mathrm{ab}}$

LGB321

A PIM1 kinase inhibitor that can regulate CXCR4 expression

\section{Agents targeting VLA-4 ( $\alpha 4 \beta 1$ )/VCAM-1 axis}

\subsection{VLA-4 antagonist}

$\mathrm{BIO5192}$

A VLA-4 antagonist

Thioridazine

$\mathrm{BOP}$

CWHM-823 and -842
An allosteric antagonist of VLA-4

A dual $\alpha 9 \beta 1 / \alpha 4 \beta 1$ integrin antagonist

VLA-4 antagonists
Single-dose CTCE-0021 alone can induce rapid mobilization; CTCE-0021 synergizes with G-CSF in mobilization.

Single-dose SCA alone can induce rapid mobilization; SCA synergizes with G-CSF in mobilization

Single-dose ATI-2341 alone induced mobilization with similar efficacy to AMD3100

Neo-r9, Neam-r9, and r9 induced robust mobilization similar to AMD3100 when used alone and showed additive effects when combined with AMD3100.

Single-dose NOX-A12 exhibits comparable mobilization effects to that of AMD3100; NOXA12 synergizes with G-CSF to enhance mobilization.

Single-dose Me6 was more effective in mobilization than AMD3100 or G-CSF alone; Me6 synergized with G-CSF in mobilization with higher efficiency than G-CSF + AMD3100.

LGB321 enhanced AMD3100-induced mobilization.
Pelus et al. [49]

Kubonishi et al. [56]

Tchernychev et al. [68]

Berchanski et al. [69]

Vater et al. [80]

Zhang et al. [85]

Müller et al. [109]
Single-dose $\mathrm{BIO} 5192$ alone induced mobilization; BIO5192 enhanced mobilization response when combined with G-CSF, AMD3100, or tGro- $\beta$.

The mobilizing ability of thioridazine was comparable to AMD3100.

Single-dose BOP alone induced rapid mobilization comparable to that induced by AMD3100; BOP synergizes with G-CSF and AMD3100 in mobilization.

Single-dose CWHM-823 or -842 induced mobilization; the combination of CWHM-823 or
Ramirez et al. [64], Cao et al. [90], Karpova et al. [107]

Chigaev et al. [70]

Cao et al. [90]

Karpova et al. [107] 
Table 1 Detailed information and mobilization efficacy of all included novel agents (Continued)

\begin{tabular}{|c|c|c|}
\hline Agent & Detailed information & HSPC mobilization efficacy \\
\hline & & -842 with tGro- $\beta$ enhanced mobilization. \\
\hline Firategrast & A VLA-4 antagonist & $\begin{array}{l}\text { Single-dose firategrast induced mobilization; the } \\
\text { combination of firategrast with } \mathrm{tGro-} \beta \text { enhanced } \\
\text { mobilization. }\end{array}$ \\
\hline \multicolumn{3}{|c|}{ 3.2 Other agents targeting VLA-4/VCAM-1 axis } \\
\hline $\begin{array}{l}\text { Anti-VCAM-1 } \\
\mathrm{Ab}\end{array}$ & Antibody of VCAM-1 & $\begin{array}{l}\text { Anti-VCAM-1 Ab alone can induce mobilization; } \\
\text { the combination of Anti-VCAM-1 Ab with G-CSF } \\
\text { increased mobilization. }\end{array}$ \\
\hline Bortezomib & $\begin{array}{l}\text { A proteasome inhibitor that can } \\
\text { inhibit transcription and expression } \\
\text { of VCAM-1 }\end{array}$ & $\begin{array}{l}\text { Single-dose bortezomib induced significant } \\
\text { mobilization; Bortezomib enhanced the } \\
\text { mobilization effect of G-CSF and AMD-3100. }\end{array}$ \\
\hline Ixazomib & $\begin{array}{l}\text { A novel proteasome inhibitor that } \\
\text { is speculated to modulate VLA4/ } \\
\text { VCAM1 axis as bortezomib }\end{array}$ & $\begin{array}{l}\text { Single-dose ixazomib can induce mobilization; } \\
\text { ixazomib synergizes with G-CSF but not } \\
\text { AMD3100 to enhance mobilization. }\end{array}$ \\
\hline
\end{tabular}

\section{References}

Karpova et al. [107]

Kikuta et al. [32], Saez et al. [84]

Ghobadi et al. [82]

Ghobadi et al. [100] AMD3100 to enhance mobilization.

\section{Heparan sulfate}

Fucoidan

A sulfated polysaccharide that can competitively displace SDF-1 from heparan sulfate proteoglycan anchors.

Fucoidan alone induced rapid HSPC mobilization. Fucoidan works synergistically with G-CSF in mobilization.

EP80031

A heparan sulfate mimetic that can compete with endogenous heparan sulfate.

Heparin

A pharmacological competitive inhibitor of heparan sulfate

\section{Agents targeting purinergic signaling}

AMP, ATP Extracellular nucleotides

DP $\quad \begin{aligned} & \text { Dipyridamole, a drug inhibiting the } \\ & \text { cellular uptake of adenosine }\end{aligned}$

ARL67156, Inhibitor of cell surface

AMPCP ectonucleotidase CD39 or CD73

\section{Agents inhibiting Cdc42 activity}

Erlotinib

An EGFR inhibitor that can reduce Cdc42 activity

ML141

A Cdc42 inhibitor

CASIN

A Cdc42 activity-specific inhibitor

\section{Agents targeting sympathetic nervous system signaling}

Desipramine, Norepinephrine reuptake inhibitors reboxetine

Adrenaline Catecholaminergic neurotransmitter

NE

Norepinephrine, catecholaminergic neurotransmitter
Single-dose EP80031 alone induced rapid mobilization with efficacy comparable to G-CSF and AMD 3100; EP80031 can act synergistically with G-CSF and AMD 3100 to mobilize HSPCs.

Heparin alone only induced modest mobilization; Saez et al. [84] heparin plus G-CSF increased the mobilization of long-term reconstituting and efficient selfrenewing cells.

Combination of AMP with DP induced significant mobilizing effects; ATP enhanced G-CSF- and

AMD 3100-induced mobilization

$D P+A M P$ induced significant mobilizing effects.

Both ARL67156 and AMPCP can enhance G-CSFand AMD 3100-induced mobilization

Erlotinib alone did not induce mobilization; erlotinib enhanced G-CSF-mediated mobilization.

ML141 alone only induced modest mobilization but played a synergistic effect in G-CSF-mediated mobilization.

Single-dose CASIN alone can induce mobilization; CASIN enhanced G-CSF- and AMD 3100-induced mobilization.

Hofer et al. [41]

Ryan et al. [67]

Chen et al. [81]

Liu et al. [108]
Frenette et al. [31], Sweeney et al. [34], Sweeney et al. [39], Albanese et al. [60]

di Giacomo et al. [72]
Hofer et al. [41], Adamiak et al. [99]

Adamiak et al. [104]

Lucas et al. [74]

mine alone did not induce mobilizati desipramine and reboxetine enhanced G-CSFinduced mobilization but did not affect AMD3100-induced mobilization.

Adrenaline alone did not induce mobilization adrenaline enhanced the mobilization efficiency of G-CSF.

NE alone can induce mobilization; NE enhanced AMD3100-induced mobilization.

\section{Agents targeting S1P signaling}


Table 1 Detailed information and mobilization efficacy of all included novel agents (Continued)

\begin{tabular}{cll}
\hline Agent & Detailed information & HSPC mobilization efficacy \\
\hline SEW2871 & A S1PR1 agonist & $\begin{array}{l}\text { SEW2871 alone did not induce mobilization; } \\
\text { administration of SEW2871 enhanced AMD3100- } \\
\text { but not G-CSF-mediated mobilization. } \\
\text { VPC01091 }\end{array}$ \\
& A selective S1PR3 antagonist & $\begin{array}{l}\text { VPC1091 alone can induce mobilization; } \\
\text { VPC01091 enhanced AMD3100-mediated } \\
\text { mobilization. }\end{array}$ \\
THI & $\begin{array}{l}\text { An inhibitor of sphingosine } \\
\text { phosphate lyase }\end{array}$ & $\begin{array}{l}\text { THI enhanced mobilization induced by G-CSF } \\
\text { and AMD3100. }\end{array}$ \\
SLM6031434 & $\begin{array}{l}\text { An inhibitor of sphingosine kinase } \\
\text { type 2 }\end{array}$ & $\begin{array}{l}\text { SLM6031434 enhanced mobilization induced by } \\
\text { G-CSF and AMD3100. }\end{array}$ \\
Anti-CD69 Ab & $\begin{array}{l}\text { An antibody of CD69 that can } \\
\text { increase S1PR1 expression }\end{array}$ & $\begin{array}{l}\text { Anti-CD69 Ab induced mobilization of the same } \\
\text { magnitude as AMD3100 but did not synergize } \\
\text { with AMD3100. }\end{array}$
\end{tabular}

\section{References}

Juarez et al. [73], Ogle et al. [96]

Ogle et al. [96]

Adamiak et al. [93]

Adamiak et al. [93]

Notario et al. [102] with AMD3100.

\section{Other agents}

CY, paclitaxel and docetaxel

Chemotherapeutic agents

PGG-glucan
ProGP

A polysaccharide

Progenipoietin-1, an agonist of both the G-CSF and FLT-3 receptors

Defibrotide

A polydeoxyribonucleotide

a-LFA-1, a-Mac- Antibody of $\beta 2$ integrin LFA-1 or Mac-1

Anti-CD49d Ab Antibody of CD49d

s-kit

A soluble form of c-kit receptor

uPAR $_{84-95}$

A derived chemotactic peptide of the cleaved forms of soluble UPAR

VTP195183

A RARa specific agonist

Anti-Notch2 Ab Antibody of Notch2

PTH

Parathyroid hormone

Tenecteplase, Thrombolytic agents microplasmin

$\mathrm{OTR}_{4120}$

$\mathrm{OTR}_{4131}$

Im-HD

Immobilized hyaluronidase

Meloxicam,

NSAIDs

indomethacin
Priming with cyclophosphamide, paclitaxel, or docetaxel induced mobilization and enhanced GCSF-induced mobilization.

Single-dose PGG-glucan alone can induce mobilization; PGG-glucan enhanced G-CSFmediated mobilization.

ProGP-mobilized cells exhibited greater spleen colony-forming activity and competitive repopulating activity than that of G-CSF.

Defibrotide alone had no mobilizing activity, addition of defibrotide significantly enhanced GCSF-induced mobilization.

The antibodies themselves had no mobilizing capacity; a-LFA-1 and a-Mac-1 increased G-CSFinduced mobilization.

5-day anti-CD49d Ab alone can induce mobilization; anti-CD49d Ab enhanced G-CSFinduced mobilization.

s-kit alone can induce mobilization; s-kit increased G-CSF-induced mobilization.

2-day UPAR $_{84-95}$ exhibited mobilization potency similar to that of 5-day G-CSF; uPAR ${ }_{84-95}$ did not act synergistically or additively with G-CSF.

VTP195183 alone did not induce mobilization; VTP195183 synergizes with G-CSF to enhance mobilization.

Single-dose anti-Notch2 Ab enhanced G-CSFand AMD3100-induced mobilization.

6-day PTH alone can induce mobilization; a combination of PTH and G-CSF showed slight additional effects.

Tenecteplase and microplasmin enhanced GCSF-induced mobilization.

Single-dose OTR 4120 or OTR OT131 $_{4}$ can induce mobilization as effectively as G-CSF and AMD3100; they synergize with G-CSF or AMD3100 in mobilization.

The native hyaluronidase and Im-HD alone did not induce significant mobilization; Im-HD enhanced G-CSF-induced mobilization.

Meloxicam or indomethacin alone can induce mobilization; meloxicam and indomethacin enhanced G-CSF- and AMD 3100-induced
Neben et al. [19], Verma et al. [29]

Ojeifo et al. [43]

Patchen et al. [26], Cramer et al. [28]

Fleming et al. [35]

Carlo-Stella et al. [38]

Velders et al. [40]

Liu et al. [45]

Nakamura et al. [46]

Selleri et al. [50]

Herbert et al. [55]

Wang et al. [98]

Brunner et al. [57]

Tjwa et al. [59]

Albanese et al. [60]

Dygai et al. [76]

Hoggatt et al. [101] 
Table 1 Detailed information and mobilization efficacy of all included novel agents (Continued)

\begin{tabular}{|c|c|c|c|}
\hline Agent & Detailed information & HSPC mobilization efficacy & References \\
\hline & & mobilization. & \\
\hline $\begin{array}{l}\mathrm{AH} 23848 \text { and } L- \\
161,982\end{array}$ & EP4 receptor antagonists & $\begin{array}{l}\text { Co-administration of AH23848 or L-161,982 with } \\
\text { G-CSF significantly enhanced mobilization. }\end{array}$ & Hoggatt et al. [101] \\
\hline UDP-G & Uridine diphosphate-glucose & $\begin{array}{l}\text { UDP-G showed comparable mobilizing ability to } \\
\text { G-CSF; the combination of UDP-G and G-CSF en- } \\
\text { hanced mobilization. }\end{array}$ & Kook et al. [79] \\
\hline FG-4497 & A HIF-1a PHD inhibitor & $\begin{array}{l}\text { FG-4497 alone did not induce mobilization; FG- } \\
4497 \text { synergizes with G-CSF and AMD } 3100 \text { to } \\
\text { enhance mobilization. }\end{array}$ & $\begin{array}{l}\text { Forristal et al. [86], Nowlan et al. [95], } \\
\text { Bisht et al. [106] }\end{array}$ \\
\hline CasNa & Sodium caseinate & $\begin{array}{l}\text { Four-dose CasNa induced significant } \\
\text { mobilization. }\end{array}$ & Santiago-Osorio et al. [87] \\
\hline SnPP & $\begin{array}{l}\text { Tin protoporphyrin IX, an inhibitor } \\
\text { of HO-1 }\end{array}$ & $\begin{array}{l}\text { SnPP significantly increased G-CSF- and AMD } \\
\text { 3100-induced HSPC mobilization. }\end{array}$ & Wysoczynski et al. [88] \\
\hline HS6101 & A small molecule lipopeptide & $\begin{array}{l}\text { Single-dose HS6101 alone can induce } \\
\text { mobilization. }\end{array}$ & Xing et al. [89] \\
\hline Dexamethasone & Glucocorticoid & $\begin{array}{l}\text { Dexamethasone enhanced AMD3100-induced } \\
\text { mobilization. }\end{array}$ & Yan et al. [92] \\
\hline Viagra & Sildenafil citrate & $\begin{array}{l}\text { Single-dose Viagra did not induce mobilization, } \\
\text { but significantly improved AMD3100-induced } \\
\text { mobilization. }\end{array}$ & Smith-Berdan et al. [110] \\
\hline CoPP & Cobalt protoporphyrin IX & $\begin{array}{l}\text { 5-day CoPP induced mobilization more efficiently } \\
\text { than G-CSF. }\end{array}$ & Szade et al. [111] \\
\hline
\end{tabular}

${ }^{a}$ Agents with superior mobilization potentials compared with G-CSF

${ }^{\mathrm{b}}$ Agents with superior mobilization potentials compared with AMD3100

Abbreviations: AMP adenosine monophosphate, ATP adenosine triphosphate, Cdc42 Cell division control protein 42, CSF1 Colony-stimulating factor 1, CXCR-4 C-X-C chemokine receptor type 4, CY cyclophosphamide, EGFR Epidermal growth factor receptor, EP4 E-proteinoid 4, G-CSF Granulocyte colony-stimulating factor, HIF-1a Hypoxia-inducible transcription factor 1a, HO-1 Heme oxygenase 1, HSPCS hematopoietic stem and progenitor cells, LFA-1 Leukocyte function antigen-1, Mac-1 macrophage antigen-1, NSAIDs nonsteroidal anti-inflammatory drugs, PHD Prolyl hydroxylase domain enzyme, PIM1 Proviral integration site for Moloney murine leukemia virus, RARa Retinoic acid receptor alpha, SDF-1 Stromal cell-derived factor-1, S1PR1 Sphingosine-1-phosphate receptor 1, S1PR3 Sphingosine-1-phosphate receptor 3, THI Tetrahydroxybutylimidazole, UPAR urokinase receptor, VCAM-1 Vascular cell adhesion molecule-1, VLA-4 Very late antigen-4

CrI 446.2-713.8), long-term SD G-CSF + reboxetine (MD 576.0, 95\% CrI 395.1-756.6), AMD3100 + VPC01091 (MD 558.7, 95\% CrI 446.6-668.9), long-term SD G-CSF + FG-4497 (MD 515.3, 95\% CrI 338.8-692.6), Me6 (MD 493.5, 95\% CrI 397.1-590.6), long-term SD G-CSF + EP80031 (MD 484.7, 95\% CrI 361.4-608.4), POL5551 (MD 429.8, 95\% CrI 259.0-600.9), long-term SD G-CSF + AMD3100 (MD 424.6, 95\% CrI 360.1487.9), AMD1300 + EP80031 (MD 417.2, 95\% CrI 306.1-530.7), and long-term LD G-CSF + meloxicam (MD 316.1, 95\% CrI 126.2, 502.4) (Fig. 3). Long-term SD G-CSF + Me6 ranked first among these regimens in regard to the ability to mobilize CFCs. AMD1300 + desipramine, Cdc42 activity-specific inhibitor (CASIN) alone, AMD3100 alone, EP80031 alone, and meloxicam alone are inferior to long-term SD G-CSF. No significant differences are identified between the other regimens and long-term SD G-CSF.

\section{LSK cells}

The number of LSK cells/ml PB was reported as primary outcome in 11 studies, which have evaluated the efficacy of 34 mobilization regimens with mice models. The network graph of all comparisons in these 11 studies is shown in Fig. 4. The results of Bayesian network metaanalysis indicate that in comparison with long-term SD G-CSF alone, 7 mobilization regimens significantly increased the number of LSK cells collected from peripheral blood, including long-term SD G-CSF + AMD3100 (MD 2577.0, 95\% CrI 2422.0-2733.0), AMD3100 + EP80031 (MD 1543.0, 95\% CrI 1385.0-1705.0), longterm SD G-CSF + EP80031 (MD 1031.0, 95\% CrI 851.71213.0), short-term SD G-CSF + AMD3100 + IL-33 (MD 766.3, 95\% CrI 576.4-960.6), long-term SD G-CSF + ML141(MD 390.7, 95\% CrI 193.2-585.9), short-term LD G-CSF + ARL67156 (MD 390.4, 95\% CrI 207.4574.4), and long-term LD G-CSF + meloxicam (MD 239.0, 95\% CrI 55.9-426.5). The MD and 95\% CrI of all included regimens are presented in forest plot in the order of median rank (Fig. 5). Long-term SD G-CSF + AMD3100 ranked first among these regimens considering this parameter since it is associated with most favorable MD and ranked first in all simulations. AMD3100 + LECT2, long-term LD G-CSF, short-term SD G-CSF, AMD3100 + IL-33, meloxicam alone, LECT2 alone, short-term LD G-CSF, and EP80031 alone are inferior to 
Table 2 Characteristics of the 21 studies included in meta-analysis

\begin{tabular}{|c|c|c|c|}
\hline Study & $\begin{array}{l}\text { Mice } \\
\text { characteristics }\end{array}$ & Experimental arm & Dose \\
\hline $\begin{array}{l}\text { Abraham } \\
\text { et al. [51] }\end{array}$ & $\begin{array}{l}\text { C57BL/6, Female, } \\
7-8 \text { weeks }\end{array}$ & $\begin{array}{l}\text { Long-term SD G-CSF; AMD3100; T-140; long-term SD G-CSF + } \\
\text { T-140; long-term SD G-CSF + AMD3100 }\end{array}$ & $\begin{array}{l}\text { G-CSF, } 2.5 \mu \mathrm{g} / \mathrm{mouse} \text { s.c. twice daily for } 4 \text { days; } \\
\text { AMD3100 } 5 \mathrm{mg} / \mathrm{kg} \text { s.c. } 2 \mathrm{~h} \text { before harvest; T-140 } 5 \\
\mathrm{mg} / \mathrm{kg} \text { s.c. } 2 \mathrm{~h} \text { before harvest }\end{array}$ \\
\hline $\begin{array}{l}\text { Kubonishi } \\
\text { et al. [56] }\end{array}$ & $\begin{array}{l}\text { C57BL/6, male } \\
\text { and female, } 7- \\
12 \text { weeks }\end{array}$ & SCA; long-term SD G-CSF; long-term SD G-CSF + SCA & $\begin{array}{l}\text { G-CSF, } 125 \mu \mathrm{g} / \mathrm{kg} \text { s.c. twice daily for } 4 \text { days; SCA, } 100 \\
\mathrm{mg} / \mathrm{kg} \text { i.v. } 30 \mathrm{~min} \text { before harvest }\end{array}$ \\
\hline
\end{tabular}
di C57BL/6 EP80031; long-term SD G-CSF + EP80031; AMD3100 + Giacomo EP80031; long-term SD G-CSF + AMD3100; long-term SD G- et al. [72] CSF + AMD3100 + EP80031

Lucas et al. [74]

C57BL/6, male, 8

weeks

Hoggatt C57BL/6

et al. [77]

\begin{tabular}{|c|c|}
\hline $\begin{array}{l}\text { Karpova } \\
\text { et al. [78] }\end{array}$ & C57BL/6 \\
\hline $\begin{array}{l}\text { Chen } \\
\text { et al. [81] }\end{array}$ & C57BL/6 \\
\hline $\begin{array}{l}\text { Ghobadi } \\
\text { et al. [82] }\end{array}$ & C57BL/6 \\
\hline $\begin{array}{l}\text { He et al. } \\
\text { [83] }\end{array}$ & $\begin{array}{l}\text { C57BL/6, 8-10 } \\
\text { weeks }\end{array}$ \\
\hline $\begin{array}{l}\text { Saez et al. } \\
{[84]}\end{array}$ & $\begin{array}{l}\text { C57BL/6, male, } \\
\text { 6-12 weeks }\end{array}$ \\
\hline $\begin{array}{l}\text { Zhang } \\
\text { et al. [85] }\end{array}$ & C57BL/6 \\
\hline $\begin{array}{l}\text { Forristal } \\
\text { et al. [86] }\end{array}$ & $\begin{array}{l}\text { C57BL/6, male, } \\
9-12 \text { weeks }\end{array}$ \\
\hline $\begin{array}{l}\text { Lu et al. } \\
\text { [91] }\end{array}$ & $\begin{array}{l}\text { C57BL/6, male, } \\
\text { 6-8 weeks }\end{array}$ \\
\hline $\begin{array}{l}\text { Karpova } \\
\text { et al. [94] }\end{array}$ & C57BL/6 \\
\hline
\end{tabular}

POL5551; HD POL5551; AMD3100

Long-term SD G-CSF; ML141; long-term SD G-CSF + ML141

Bortezomib; long-term SD G-CSF; AMD3100; long-term SD GCSF + Bortezomib; AMD3100 + Bortezomib

10d-FLT-3L; short-term SD G-CSF + AMD3100; 10d-FLT-3L + AMD3100

Long-term SD G-CSF; long-term SD G-CSF + heparin; G-CSF + Anti-VCAM-1 Ab; AMD310; heparin; AMD3100 + heparin

Me6; AMD3100; long-term SD G-CSF; long-term SD G-CSF + AMD3100; long-term SD G-CSF + Me6

Long-term SD G-CSF; long-term SD G-CSF + AMD3100; longterm SD G-CSF + FG-4497; long-term SD G-CSF + AMD3100 + FG-4497

LECT2; AMD3100; AMD3100 + LECT2

HD POL5551; 14d-HD POL5551; 14d-HD AMD3100; 14dALT1188; 14d-HD POL5551 + HD AMD3100; 14d-HD POL5551 + CWHM-823

Ogle et al. C57BL/6, male, [96] 8-12 weeks

Adamiak C57BL/6, 4-6 et al. [104] weeks

Alt et al. C57BL/6, Male, [105] 6-10 weeks

Bisht et al. C57BL/6, male, [106] 8-9 weeks
VPC01091; AMD3100; AMD3100 + VPC01091

Short-term LD G-CSF; AMD3100; short-term LD G-CSF + ARL67156; AMD3100 + ARL67156; short-term LD G-CSF + AMPCP; AMD3100 + AMPCP

Short-term SD G-CSF; AMD3100; short-term SD G-CSF + AMD3100; IL-33; short-term SD G-CSF + IL-33; AMD 3100 + IL33; short-term SD G-CSF + AMD3100 + IL-33

Short-term SD G-CSF; short-term SD G-CSF + FG-4497
EP80031, 15 mg/kg i.v. 1 h before harvest; G-CSF, 2.5 $\mu \mathrm{g} / \mathrm{mouse}$ s.c. twice daily for 4 days; AMD1300, 5 mg/ $\mathrm{kg}$ s.c. $1 \mathrm{~h}$ before harvest

G-CSF, $125 \mu \mathrm{g} / \mathrm{kg}$ s.c. twice daily for 4 days; AMD $3100,5 \mathrm{mg} / \mathrm{kg}$ s.c. $1 \mathrm{~h}$ before collection; desipramine, $10 \mathrm{mg} / \mathrm{kg} /$ day i.p. for 8 days; reboxetine, $5 \mathrm{mg} / \mathrm{kg} /$ day i.p. for 8 days

G-CSF, 50 mg/kg s.c. twice daily for 4 days; AMD 3100, $5 \mathrm{mg} / \mathrm{kg}$ i.p. $1 \mathrm{~h}$ before collection; meloxicam, 0.5-12 $\mathrm{mg} / \mathrm{kg}$ s.c. for 4 days; indomethacin, $0.5-2.5 \mathrm{mg} / \mathrm{kg}$ s.c. twice daily for 4 days; $\mathrm{AH} 23848,10 \mu \mathrm{g}$ per mouse i.p. for 4 days; L-161,982, $10 \mu \mathrm{g}$ per mouse i.p. for 4 days

POL5551, 5 or 100 (HD) mg/kg i.p. 2 or $4 \mathrm{~h}$ before harvest; AMD3100, 5 mg/kg i.p. 1 h before harvest.

G-CSF, $200 \mu \mathrm{g} / \mathrm{kg} /$ day s.c. for 5 days; ML141, $10 \mu \mathrm{g} /$ $\mathrm{kg} /$ day i.p. for 5 days

Bortezomib, a single dose of $0.8 \mathrm{mg} / \mathrm{kg}$ i.v.; G-CSF, 250 $\mu \mathrm{g} / \mathrm{kg} /$ day s.c. for 4 days; AMD3100, 5 mg/kg s.c.

FLT-3L, 350 mg/kg/day i.p. for 10 days; G-CSF, 150 mg/ $\mathrm{kg} /$ day i.p. for 5 days; AMD3100, 5 mg/kg i.p. $1 \mathrm{~h}$ before harvest

G-CSF, $125 \mu \mathrm{g} / \mathrm{kg}$ s.c. twice for 4 days; heparin, $100 \mathrm{U}$ i.p. $1 \mathrm{~h}$ before harvest; Anti-VCAM-1 Ab, 2 mg/kg/day i.v. for 3 days; AMD3100, 5 mg/kg s.c. 1 h before harvest

Me6, 5 mg/kg s.c. $12 \mathrm{~h}$ before harvest; AMD3100, $5 \mathrm{mg} / \mathrm{kg}$ s.c. $1 \mathrm{~h}$ before harvest; G-CSF, $2.5 \mu \mathrm{g}$ per mouse s.c. twice daily for 4 days

G-CSF, 125 mg/kg s.c. twice daily for 4 days; AMD3100, $5 \mathrm{mg} / \mathrm{kg}$ s.c. $1 \mathrm{~h}$ before harvest; FG-4497, $20 \mathrm{mg} / \mathrm{kg} /$ day i.p. for 3 days

LECT2, $300 \mu \mathrm{g} / \mathrm{kg} /$ day s.c. for 5 days; AMD 3100, 5 $\mathrm{mg} / \mathrm{kg}$ s.c. $1 \mathrm{~h}$ before collection

POL 5551, 100mg/kg i.p. as a single dose or as continuous infusion for 2 weeks via subcutaneously implanted pumps; ALT1188, 33 mg/kg i.p. as a single injection or as continuous infusion for 2 weeks; AMD3100, $20 \mathrm{mg} / \mathrm{kg}$ i.p. as a single injection or as continuous infusion for 2 weeks; CWHM-823, 3 mg/kg i.p.

AMD3100 5mg/kg i.p. 1.5 h before harvest; VPC01091 $5 \mathrm{mg} / \mathrm{kg}$ i.p. $1.5 \mathrm{~h}$ before harvest

G-CSF, $100 \mu \mathrm{g} / \mathrm{kg} /$ day s.c. for 3 days; AMD 3100, 5 $\mathrm{mg} / \mathrm{kg}$ i.p. $1 \mathrm{~h}$ before collection; ARL67156, $2 \mathrm{mg} / \mathrm{kg}$ i.p.; $A M P C P, 4 \mathrm{mg} / \mathrm{kg}$ i.p.

G-CSF, $200 \mu \mathrm{g} / \mathrm{kg} /$ day s.c. for 3 days; AMD 3100, 5 $\mathrm{mg} / \mathrm{kg}$ i.p. $1 \mathrm{~h}$ before collection; IL-33, 0.04 mg/kg/ day i.p. for 3 days

G-CSF, $125 \mu \mathrm{g} / \mathrm{kg}$ s.c. twice daily for 2 days; FG-4497, $20 \mathrm{mg} / \mathrm{kg} /$ day i.p. for 3 days; 
Table 2 Characteristics of the 21 studies included in meta-analysis (Continued)

\begin{tabular}{|c|c|c|c|}
\hline Study & $\begin{array}{l}\text { Mice } \\
\text { characteristics }\end{array}$ & Experimental arm & Dose \\
\hline $\begin{array}{l}\text { Liu et al. } \\
\text { [108] }\end{array}$ & C57BL/6 & CASIN; AMD3100; CASIN + AMD3100 & $\begin{array}{l}\text { CASIN, } 1.2 \mathrm{mg} / \mathrm{kg} \text { i.v. } 2 \mathrm{~h} \text { before harvest; AMD3100, } \\
5 \mathrm{mg} / \mathrm{kg} \text { i.p. } 2 \mathrm{~h} \text { before harvest. }\end{array}$ \\
\hline $\begin{array}{l}\text { Müller } \\
\text { et al. [109] }\end{array}$ & C57BL/6 & AMD3100; AMD3100 + LGB321 & AMD3100, 5 mg/kg s.c.; LGB321, 100 mg/kg s.c. \\
\hline $\begin{array}{l}\text { Fang et al. } \\
{[112]}\end{array}$ & C57BL/6 & $\begin{array}{l}\text { HF51116; AMD 3100; long-term SD G-CSF; G-CSF + HF51116; } \\
\text { long-term SD G-CSF + AMD } 3100\end{array}$ & 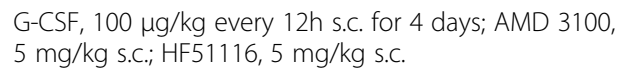 \\
\hline
\end{tabular}

Abbreviations: ALT1188 a small molecule CXCR4 antagonist; AMPCP an inhibitor of cell surface ectonucleotidase CD73; ARL67156 an inhibitor of cell surface ectonucleotidase CD39; CFCs colony-forming cells; CASIN cell division control protein 42 (Cdc42) activity-specific inhibitor; CWHM-823 small molecule very late antigen 4 (VLA4) antagonist; EP80031 synthetic octo-saccharides, a heparan sulfate mimetic; FG-4497 hypoxia-inducible transcription factor prolyl hydroxylase domain enzymes inhibitor; FLT-3L fms-like tyrosine kinase-3 ligand; G-CSF granulocyte colony-stimulating factor; HD high dose; HF51116 a new CXCR4 antagonist; IL-33 interleukin 33; LD low dose; LGB321 Proviral integration site for Moloney murine leukemia virus (PIM) kinase inhibitor; $L S K$ cells Lin ${ }^{-}$Sca ${ }^{+}{ }^{~ K i t}{ }^{+}$cells; Me6 Me6TREN, Tris[2-(dimethylamino)ethyl]amine); ML141 cell division control protein 42 (Cdc42) inhibitor; PEGLip-G-CSF pegylated liposome formulated granulocyte colony-stimulating factor; POL5551 a peptidic CXCR4 antagonist; SCA sulfated colominic acid; SD standard dose; T-140 4F-benzoyl-TN14003, a highly selective CXCR4 antagonist; VPC01091 a selective sphingosine-1-phosphate receptor 3 antagonist

G-CSF in regard to the ability of mobilizing LSK cells into blood. No significant differences are identified between the other regimens and long-term SD G-CSF.

\section{Long-term repopulating ability}

Although the number of CFCs and LSK cells are the most commonly used outcomes to evaluate HSPC mobilization efficiency, enriched cell subsets such as CFCs and LSK cells do not measure long-term reconstituting HSCs, and additional markers such as fms-like tyrosine kinase-3 (Flt3) and signaling lymphocyte activation molecule (SLAM) CD150 were used to identify LSK subsets and assess the mobilization of self-renewing
HSCs and long-term HSCs (LT-HSCs) [114]. The mobilization of different LSK subsets were examined in 12 studies, and the results are summarized in Supplementary Table 3. In brief, combination of desipramine, meloxicam, hypoxia-inducible transcription factor $1 \alpha$ (HIF-1 $\alpha$ ) prolyl hydroxylase domain enzyme (PHD) inhibitors (FG-4497, PHI-1, or PHI-2), the dual $\alpha 9 \beta 1 / \alpha 4 \beta 1$ integrin antagonist BOP, Viagra, new CXCR4 antagonist HF51116, and colony-stimulating factor $1 \mathrm{Fc}$ fusion protein (CSF1-Fc) significantly increased the mobilization of LSKF cells $\left(\mathrm{Lin}^{-} \mathrm{Sca}-1^{+} \mathrm{c}-\mathrm{kit}^{+} \mathrm{Flt} 3^{-}\right.$cells), SLAM LSK cells ( $\mathrm{Lin}^{-} \mathrm{Sca}-1^{+} \mathrm{c}-\mathrm{kit}^{+} \mathrm{CD} 48^{-} \mathrm{CD} 150^{+}$cells), or LT-HSCs compared with G-CSF alone. The truncated form of

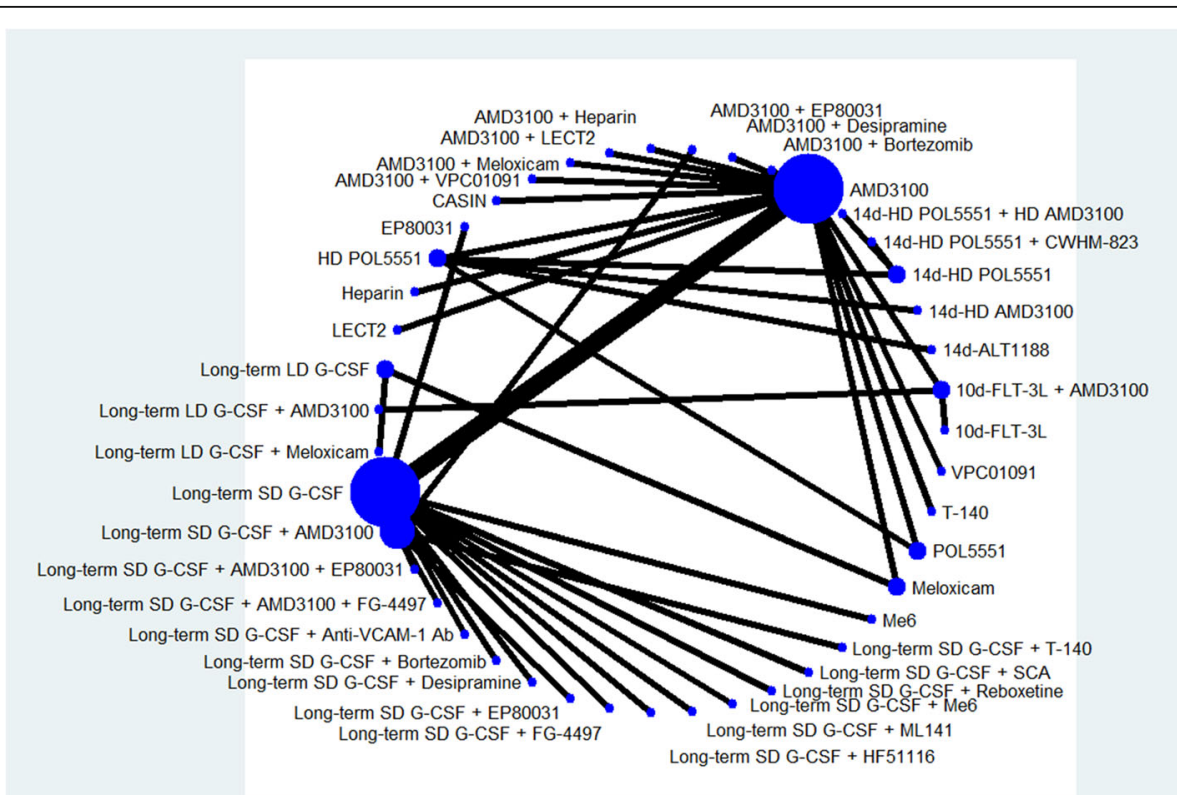

Fig. 2. Network graph for total CFCs. The network graph of all comparisons in the 21 studies that have data about total colony-forming cells (CFCs) per milliliter of peripheral blood (/ml PB). Each node represents a mobilization regimen, while each line represents a direct comparison between regimens, with the thickness reflecting the number of available direct comparisons. All included regimens are described in the supplementary materials 


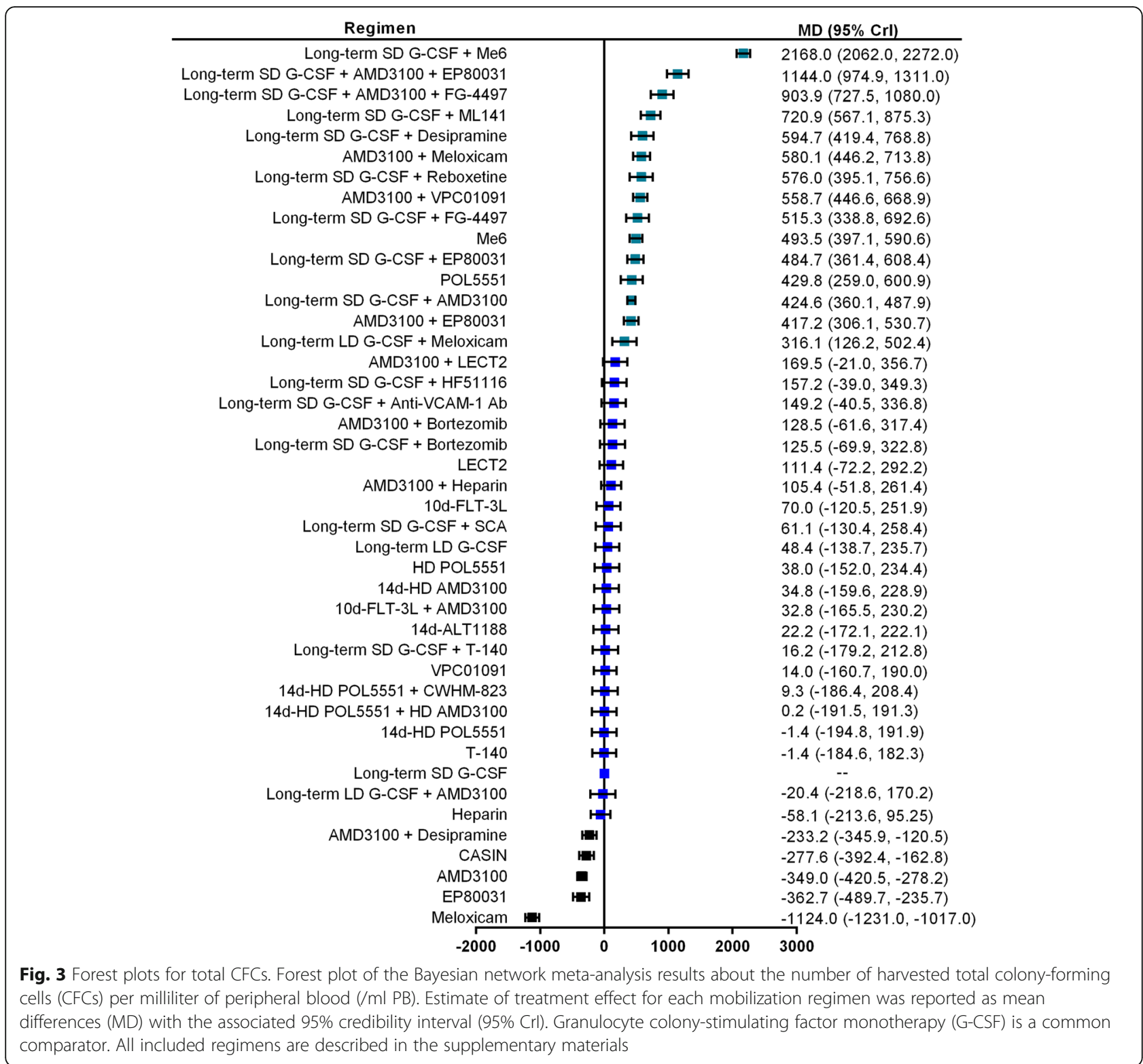

chemokine GRO $\beta$ (tGRO $\beta$ ) plus AMD3100, Cobalt protoporphyrin IX (CoPP), mobilized higher levels of SLAM LSK cells than G-CSF.

To further assess the mobilization of long-term repopulating HSCs, in vivo transplantation experiments were performed in 49 studies. The characteristics and results of these 49 studies are reviewed in Supplementary Table 4 . In summary, lethally irradiated recipient mice received mobilized PB cells from donor mice with or without competitive cells, and the long-term repopulating ability are assessed by the survival of recipients and the long-term reconstitution donor-derived cells at different time point (usually in at months after transplantation). Furthermore, serial transplantation analysis was performed via transplanting BM cells from primary recipients to secondary or tertiary recipients to assess the long-term repopulating and self-renewing capacity of mobilized cells in 20 studies. Results indicate that the combination of new mobilization agents (including FLT3L, MIP-1 $\alpha$, IL-8, PEG-rHuMGDF, SB-251353, s-kit, AMD3100, T-140, tGRO $\beta$, VTP195183, SCA, erlotinib, EP80031, meloxicam, UDP-G, Anti-VCAM-1 Ab, heparin, Me6, HF51116, and CSF1-Fc) significantly increased the mobilization of long-term repopulating HSCs compared with G-CSF alone. In addition, the combination of BOP, BIO5192, SEW2871, VPC01091, LGB321, or Viagra with AMD3100 enhanced the mobilization of long-term repopulating HSCs compared with AMD3100 alone. Moreover, cells mobilized by LECT2, POL5551, UDP-G, or CoPP alone showed 


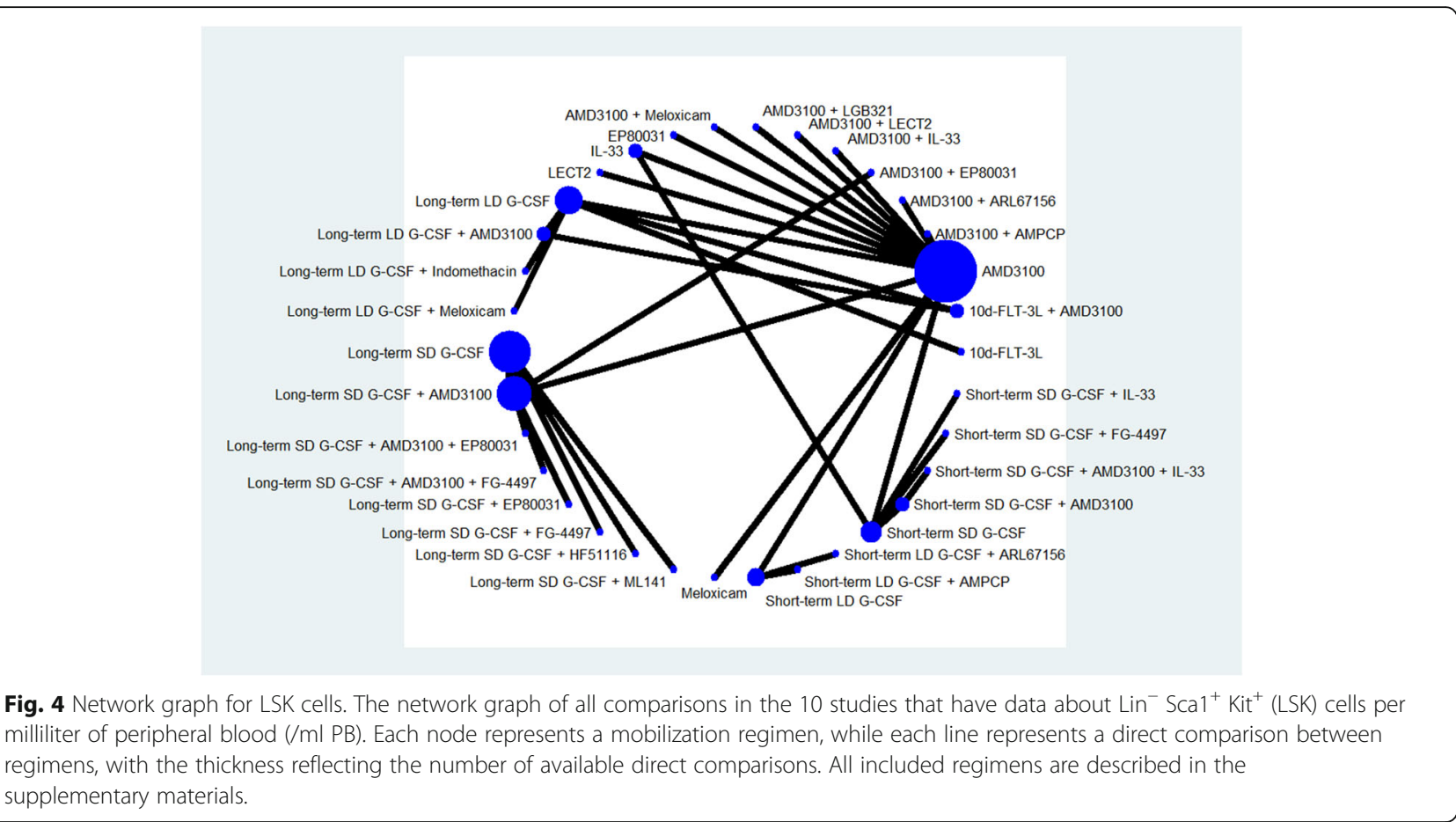

superior long-term repopulating capacity than those mobilized by G-CSF, whereas cells mobilized by Me6, CasNa, or CASIN alone showed superior long-term repopulating capacity than those mobilized by AMD3100.

\section{Discussion}

This work reviewed the efficacy of 94 new HSC mobilization agents from 95 preclinical studies. In addition, we included 21 studies using the poor mobilizer model-C57BL/6 mice for network metaanalysis and compared the efficacy of 57 mobilization regimens. We identified several promising regimens with great HSC mobilization efficacy, including long-term SD G-CSF + Me6, long-term SD G-CSF + AMD3100 + EP80031, long-term SD G-CSF + AMD3100 + FG-4497, long-term SD G-CSF + ML141, long-term SD G-CSF + desipramine, AMD3100 + meloxicam, long-term SD GCSF + reboxetine, AMD3100 + VPC01091, long-term SD G-CSF + FG-4497, Me6, POL5551, long-term SD GCSF + AMD3100, long-term LD G-CSF + meloxicam, AMD3100 + EP80031, long-term SD G-CSF + EP80031, short-term SD G-CSF + AMD3100 + IL-33, and shortterm LD G-CSF + ARL67156.

To our best of knowledge, this study is the first network meta-analysis that compared the efficacy of different HSC mobilization regimens with data from preclinical studies. We provide a comprehensive summary of new mobilization agents that have been investigated in mice models. The efficacy of these agents alone or in combination with other agents was indirectly compared via network meta-analysis. Moreover, we ranked all of the investigated regimens based on their ability to mobilize HSCs into blood stream. We identified several promising agents and regimens that have the most potent mobilizing capacity. The majority of mobilization regimens that show great improvements over G-CSF are combined regimens containing both G-CSF and new mobilization agents. Although these regimens would be unlikely to reduce severe adverse events, they provide a perspective that the incorporation of new agents could reduce the incidences of G-CSF-related adverse events through reducing the doses of G-CSF that required to mobilization sufficient HSCs since they can synergistically enhance the G-CSF-mediated mobilization. In addition, we identified several agents showed superior mobilization potential than G-CSF even when used alone, such as Me6 and POL5551. It is worth further investigation that whether these agents could reduce mobilizationrelated toxicity compared with G-CSF.

Among the new agents, EP80031, Me6, FG-4497, and ML141 significantly improved the efficiency of G-CSFinduced HSC mobilization. EP80031 is a synthetic octosaccharide mimicking the structure of heparan sulfate. A single dose of EP80031 (15mg/kg, intravenously injection) could lead to rapid and prominent mobilization of hematopoietic stem and progenitor cells (HSPCs), and the combination of EP80031 with G-CSF and AMD3100 resulted in 3-fold increase in the number of LSK cells and total CFCs [72]. In addition, HSCs mobilized with the regimen G-CSF + 


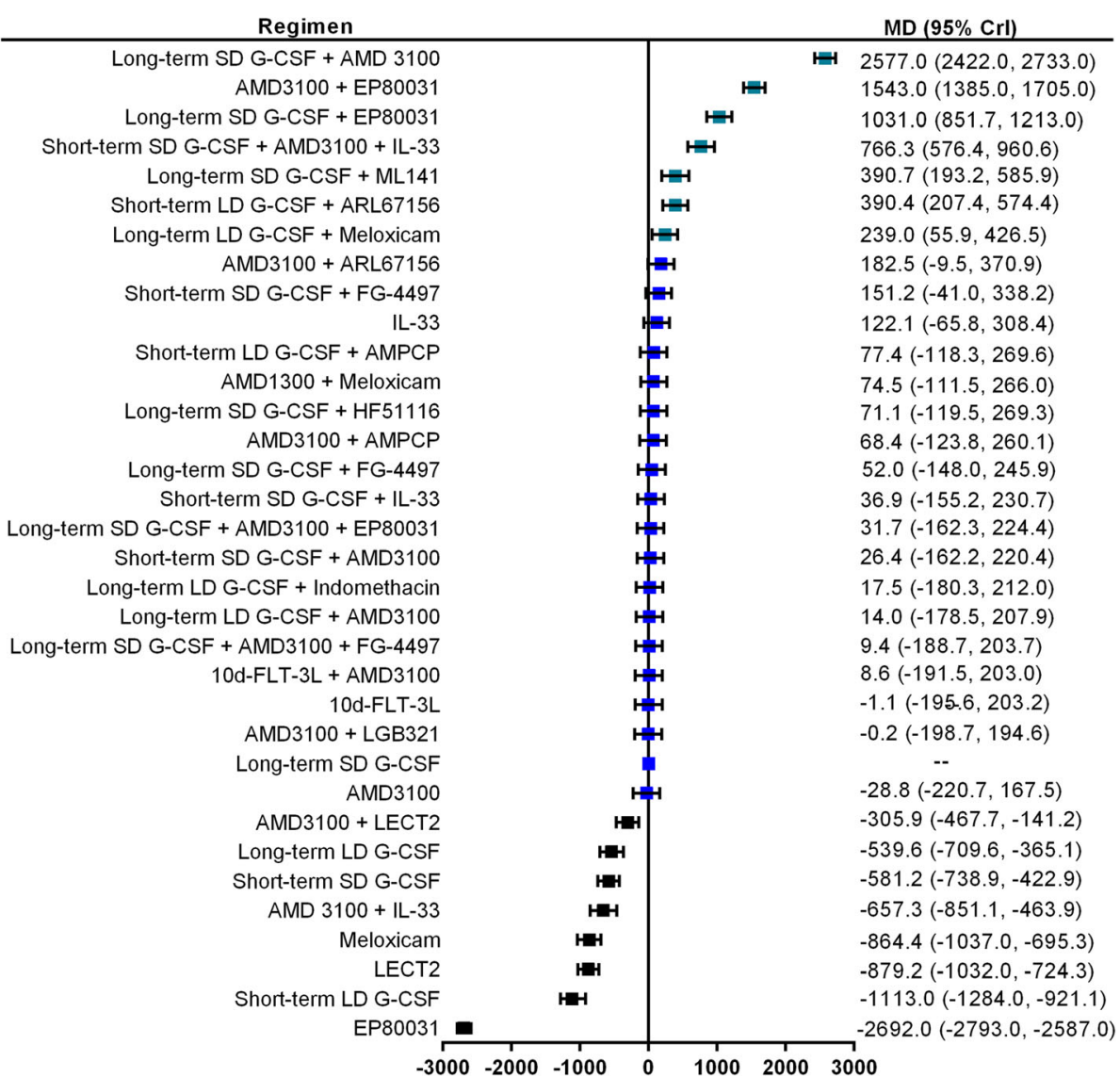

Fig. 5 Forest plots for LSK cells. Forest plot of the Bayesian network meta-analysis results about the number of harvested $\mathrm{Lin}^{-} \mathrm{Sca} 1^{+} \mathrm{Kit}^{+}(\mathrm{LSK})$ cells per milliliter of peripheral blood (/ml PB). Estimate of treatment effect for each mobilization regimen was reported as mean differences (MD) with the associated $95 \%$ credibility interval (95\% Crl). Granulocyte colony-stimulating factor monotherapy (G-CSF) is a common comparator. All included regimens are described in the supplementary materials

AMD3100 + EP80031 are associated with enhanced hematopoietic reconstitution [72]. Me6 is a small molecule that was screened from a group of chemicals by Zhang et al. and has been proved to have robust ability of mobilizing HSPCs [85]. The combination of Me6 and G-CSF (G$\mathrm{CSF}+\mathrm{Me6}$ ) resulted in remarkable increase in the number of total CFUs, moreover, it is suggested that Me6-mobilized HSCs are associated with greater long-term repopulating capacity and more efficient engraftment [85]. FG-4497 is a prolyl hydroxylase inhibitor that could enhance HSC mobilization through stabilizing the hypoxia-inducible transcription factor-1 $\alpha$ (HIF-1 $\alpha)$ protein [106]. The addition of FG-4497 significantly increased the mobilization of HSPCs induced by G-CSF [86, 106]. In addition, FG-4497 exerts protective effects in ischemia-induced kidney injury and high-dose irradiation-induced BM failure [115]. ML141 is an inhibitor of cell division control protein 42 (Cdc42). The mobilization effect of ML141 is modest, but ML141 could synergistically enhance G-CSF-mediated mobilization of LSK cells and CFCs in mice model [81]. Taking our results of meta-analysis together into consideration, G-CSF +
AMD3100 + EP80031, G-CSF + Me6, G-CSF + FG-4497, and G-CSF + ML141 are new promising mobilization regimens that could significantly increase the quantity of HSCs in $\mathrm{PB}$ without interfering their functions. However, the safety profiles of these new agents remain unclear. Further studies are required to determine the efficacy and safety of these potential regimens in human before applied in clinical practice.

In addition, we established the favorable efficacy of GCSF and AMD3100 in HSC mobilization, which has been verified by clinical trials. AMD3100, also known as plerixafor, is an antagonist of the chemokine receptor CXCR4 that could rapidly induce the mobilization of stem cells through antagonizing the interaction of CXCR4 and stromal cell-derived factor-1 $\alpha$ (SDF-1 $\alpha$ ) [116]. Multiple studies have demonstrated that AMD3100 alone mobilized lower numbers of HSCs compared with G-CSF, but the addition of AMD3100 dramatically increased the G-CSF-induced mobilization of HSCs both in mice models and non-human primates' model [117, 118]. Our results from network meta- 
analyses indicated that G-CSF in combination of AMD3100 not only significantly increased the number of LSK cells, but also increased total CFCs. Despite we only pooled data from murine models, which are different from human in regard to physiological conditions, our conclusions are consistent with that obtained from clinical studies in human beings. A group of randomized controlled trials (RCTs) have demonstrated that G-CSF in combination of AMD3100 led to higher rates of successful mobilization and increased the total collection of HSCs without increasing the risk of severe adverse events in patients with non-Hodgkin's lymphoma (NHL) and multiple myeloma (MM) [119-121]. Moreover, it is suggested that AMD3100-mobilized cell products are associated with greater capacity to repopulate the marrow and potential of protecting against graft-versus-host disease due to an enrichment of regulatory T cells (GVHD) $[118,122]$. AMD3100 has been approved for HSC mobilization and subsequent autologous transplantation in patients with NHL and MM [123]. Therefore, before the efficacy and safety of new regimens in human were well established, G-CSF in combination with AMD3100 remains the most efficient and safe regimens in patients with high risk of mobilization failure. Although G-CSF plus AMD3100 significantly improved mobilization efficiency compared with G-CSF alone, two well-designed RCTs indicated that successful rate of achieving optimal target with G-CSF plus AMD3100 is only $59.3 \%$ in NHL patients and $75.7 \%$ in MM patients [119, 120]. Therefore, we speculate that almost $25-40 \%$ of patients with high risk of mobilization failure would still benefit from new mobilization regimens.

Nevertheless, there are some limitations in this study. Firstly, we integrated evidences from animal models. It is suggested that HSC mobilization is evolutionarily conserved from mice to humans, so mice models also represent a valuable experimental system for investigating the efficacy and mechanisms of mobilization regimens $[67,81]$. Even so, animal model could not completely simulate the physiological condition of human; hence, the translation of our results integrated from preclinical studies to human should be in cautions. Future clinical trials are needed for validation these regimens in human. Secondly, our meta-analysis did not include safety outcomes. Most of the included studies did not provide information about toxicity, and the toxicity data collected from animal experiments are hard to be pooled with metaanalysis. Further studies are required to compare the safety of these new mobilization regimens. Thirdly, the results of meta-analysis may be confounded by the heterogeneity in mice gender since it was reported that male mice have better mobilization outcome compared with female mice [114]. It is impractical to perform subgroup analysis based on animal gender since most of the studies did not report the gender of mice and some studies included both male and female mice. However, since the network meta-analyses were performed with wellestablished methods and the most efficacious regimens are associated with robust MD values, we believe that the effects of these differences are minimal. Last but not least, there is a big gap between our results and translational medicine since the lack of data from human systems, but we think this study may contribute to the translation of basic research results into clinical investigations through providing comprehensive review of new promising mobilization regimens and related mechanisms.

\section{Conclusions}

In summary, this study identified several promising mobilization agents and regimens that significantly increased the mobilization of HSCs compared with the conventional agent G-CSF alone. We think that our results can provide important perspectives for future researches.

\section{Abbreviations}

ALT1188: A small molecule CXCR4 antagonist; AMD3100: A C-X-C chemokine receptor type 4 (CXCR4) antagonist; AMP: Adenosine monophosphate; ATP: Adenosine triphosphate; BOP: A dual a9 $31 / a 4 \beta 1$ antagonist; BM: Bone marrow; CFCs: Colony-forming cells; CFUs: Colony-forming units; CASIN: Cell division control protein 42 (Cdc42) activity-specific inhibitor; CasNa: Sodium caseinate; CY: Cyclophosphamide; DFS: Disease-free survival; CWHM823: Small molecule very late antigen 4 (VLA4) antagonist; Crl: Credibility interval; DIC: Deviance information criterion; EP80031: Synthetic octosaccharides, a heparan sulfate mimetic; FG-4497: Hypoxia-inducible transcription factor prolyl hydroxylase domain enzymes inhibitor; FLT-3L: fmslike tyrosine kinase-3 ligand; G-CSF: Granulocyte colony-stimulating factor; HF51116: A new CXCR4 antagonist; HSCs: Hematopoietic stem cells; HSCT: Hematopoietic stem cell transplantation; HSPCs: Hematopoietic stem and progenitor cells; LD: Low dose; LFA-1: Leukocyte function antigen-1; LGB321: Proviral integration site for Moloney murine leukemia virus (PIM)

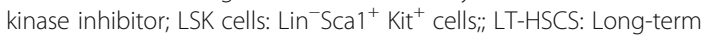
hematopoietic stem cells; MD: Mean differences; Mac-1: Macrophage antigen-1; Me6: Me6TREN, Tris[2-(dimethylamino)ethyl]amine); MIP2: Macrophage inflammatory protein-2; ML141: Cell division control protein 42 (Cdc42) inhibitor; NA: Not applicable; Neam-r9: Nona-d-arginine-neamine conjugate; Neo-r9: Nona-d-arginine-neomycin conjugate; NOX-A12: A

PEGylated mirror-image oligonucleotide that binds to CXCL12 and inhibits CXCL12 signaling; NSAIDs: Nonsteroidal anti-inflammatory drugs; OS: Overall survival; PB: Peripheral blood; PBS: Phosphate-buffered saline; PBSCs: Peripheral blood stem cells; PEGLip-G-CSF: Pegylated liposome formulated granulocyte colony-stimulating factor; POL5551: A peptidic CXCR4 antagonist; RARa: Retinoic acid receptor alpha; rhPIGF-1: Recombinant human placental growth factor-1; r9: N-a-acetyl-nona-D-arginine;

SCA: Sulfated colominic acid; SD: Standard dose; SDF-1a: Stromal cell-derived factor-1a; SLAM LSK cells: Lin $^{-}$Sca- $1^{+}{ }^{-}-$kit $^{+}$CD $48^{-}$CD150 ${ }^{+}$cells; SYRC LE: SYstematic Review Centre for Laboratory animal Experimentation; S1PR1: Sphingosine-1-phosphate receptor 1; S1PR3: Sphingosine-1phosphate receptor 3; THI: Tetrahydroxybutylimidazole; T-140: 4F-benzoylTN14003, a short-modified peptide, a highly selective CXCR4 antagonist; UPAR: Urokinase receptor; VCAM-1: Vascular cell adhesion molecule-1; VLA4: Very late antigen-4; VPC01091: A selective sphingosine-1-phosphate receptor 3 antagonist 


\section{Supplementary Information}

The online version contains supplementary material available at https://doi. org/10.1186/s13287-021-02379-6

Additional file 1: Supplementary Table 1. Characteristics of the 95 studies included for review.

Additional file 2: Supplementary Table 2. Risk of bias assessment using the SYRCLE tool.

Additional file 3: Supplementary Table 3. Results about the mobilization of different LSK subsets.

Additional file 4: Supplementary Table 4. Characteristics and results of in vivo transplantation experiments.

\section{Acknowledgements}

Not applicable.

\section{Authors' contributions}

SNX and JPC designed and supervised the study. CXL and XL performed database searching, study selection, data extraction, statistical analyses, and manuscript writing. LW and SNX contributed to data checking and manuscript revision. GXW, XTH, YLZ, YNM, MLX, YNS, YRH, ZH, QYS, HL, and $\mathrm{YH}$ helped with data extraction, data checking, and statistical analyses. All authors read and approved the final manuscript.

\section{Funding}

This work is supported by the Military Logistics Project (BWS13C018) and the National Natural Science Foundation of China (NSFC 81800172). The funding had no role in the study design, data collection, data analysis, data interpretation, or manuscript writing.

\section{Availability of data and materials}

All supporting data are included in the article and its additional files.

\section{Declarations}

Ethics approval and consent to participate

Not applicable.

\section{Consent for publication}

Not applicable.

\section{Competing interests}

The authors declare that they have no competing interests.

\section{Author details}

'Center for Hematology, Southwest Hospital, Third Military Medical University, \#30 Gaotanyan Street, Shapingba District, Chongqing 400038, China. ${ }^{2}$ Key Laboratory of Cancer Immunotherapy of Chongqing, Chongqing, China. ${ }^{3}$ Department of Hematology, The First Affiliated Hospital of Chongqing Medical University, Chongqing, China. ${ }^{4}$ Department of Health Statistics, Third Military Medical University, Chongqing, China. ${ }^{5}$ Institute of Infectious Disease, Southwest Hospital, Third Military Medical University, \#30 Gaotanyan Street, Shapingba District, Chongqing 400038, China.

Received: 14 January 2021 Accepted: 10 May 2021

Published online: 29 May 2021

References

1. Chabannon C, Kuball J, Bondanza A, Dazzi F, Pedrazzoli P, Toubert A, et al, Hematopoietic stem cell transplantation in its 60s: A platform for cellular therapies. Sci Transl Med. 2018;10(436):eaap9630.

2. Passweg JR, Baldomero H, Bader P, Bonini C, Cesaro S, Dreger $P$, et al. Hematopoietic SCT in Europe 2013: recent trends in the use of alternative donors showing more haploidentical donors but fewer cord blood transplants. Bone Marrow Transplant. 2015;50(4):476-82. https://doi.org/10.1 038/bmt.2014.312

3. Shimosato Y, Tanoshima R, Tsujimoto SI, Takeuchi M, Shiba N, Kobayashi T, et al. Allogeneic bone marrow transplantation versus peripheral blood stem cell transplantation for hematologic malignancies in children: a systematic review and meta-analysis. Biol Blood Marrow Transplant. 2020;26(1):88-93.

4. Yu X, Liu L, Xie Z, Dong C, Zhao L, Zhang J, et al. Bone marrow versus peripheral blood as a graft source for haploidentical donor transplantation in adults using post-transplant cyclophosphamide-a systematic review and meta-analysis. Crit Rev Oncol Hematol. 2019;133:120-8. https://doi.org/10.1 016/j.critrevonc.2018.05.017.

5. Amouzegar A, Dey BR, Spitzer TR. Peripheral blood or bone marrow stem cells? Practical considerations in hematopoietic stem cell transplantation. Transfusion medicine reviews. 2019;33(1):43-50. https://doi.org/10.1016/j. tmrv.2018.11.003.

6. Bonig H, Papayannopoulou T. Hematopoietic stem cell mobilization: updated conceptual renditions. Leukemia. 2013:27(1):24-31.

7. Bensinger W, DiPersio JF, McCarty JM. Improving stem cell mobilization strategies: future directions. Bone Marrow Transplant. 2009:43(3):181-95. https://doi.org/10.1038/bmt.2008.410

8. To LB, Levesque JP, Herbert KE. How I treat patients who mobilize hematopoietic stem cells poorly. Blood. 2011;118(17):4530-40. https://doi. org/10.1182/blood-2011-06-318220.

9. Pulsipher MA, Chitphakdithai P, Miller JP, Logan BR, King RJ, Rizzo JD, et al Adverse events among 2408 unrelated donors of peripheral blood stem cells: results of a prospective trial from the National Marrow Donor Program. Blood. 2009;113(15):3604-11. https://doi.org/10.1182/blood-2008-08-175323.

10. Halter J, Kodera Y, Ispizua AU, Greinix HT, Schmitz N, Favre G, et al. Severe events in donors after allogeneic hematopoietic stem cell donation. Haematologica. 2009;94(1):94-101. https://doi.org/10.3324/haematol.13668.

11. Cashen AF, Lazarus HM, Devine SM. Mobilizing stem cells from normal donors: is it possible to improve upon G-CSF? Bone Marrow Transplant. 2007;39(10):577-88. https://doi.org/10.1038/sj.bmt.1705616.

12. Roberts AW, Foote S, Alexander WS, Scott C, Robb L, Metcalf D. Genetic influences determining progenitor cell mobilization and leukocytosis induced by granulocyte colony-stimulating factor. Blood. 1997:89(8):273644. https://doi.org/10.1182/blood.V89.8.2736

13. Xing Z, Ryan MA, Daria D, Nattamai KJ, Van Zant G, Wang L, et al. Increased hematopoietic stem cell mobilization in aged mice. Blood. 2006;108(7): 2190-7.

14. Gheibi S, Mahmoodzadeh A, Kashfi K. Data extraction from graphs using Adobe Photoshop: applications for meta-analyses. Int J Endocrinol Metab. 2019;17(4):e95216.

15. Hooijmans CR, Rovers MM, de Vries RB, Leenaars M, Ritskes-Hoitinga M, Langendam MW. SYRCLE's risk of bias tool for animal studies. BMC Med Res Method. 2014;14(1):43. https://doi.org/10.1186/1471-2288-14-43.

16. Dias S, Welton NJ, Sutton AJ, Ades AE. NICE DSU Technical Support Document 2: a generalised linear modelling framework for pairwise and network meta-analysis of randomised controlled trials. 2011; last updated April 2014; available from http://www.nicedsu.org.uk. Accessed 19 May 2016.

17. Skoetz N, Trelle S, Rancea M, Haverkamp H, Diehl V, Engert A, et al. Effect of initial treatment strategy on survival of patients with advanced-stage Hodgkin's lymphoma: a systematic review and network meta-analysis. Lancet Oncol. 2013;14(10):943-52. https://doi.org/10.1016/S1470-2045(13 )70341-3.

18. Hutton B, Salanti G, Caldwell DM, Chaimani A, Schmid CH, Cameron C, et al. The PRISMA extension statement for reporting of systematic reviews incorporating network meta-analyses of health care interventions: checklist and explanations. Ann Intern Med. 2015;162(11):777-84. https://doi.org/10. 7326/M14-2385

19. Neben S, Marcus K, Mauch P. Mobilization of hematopoietic stem and progenitor cell subpopulations from the marrow to the blood of mice following cyclophosphamide and/or granulocyte colony-stimulating factor. Blood. 1993;81(7):1960-7. https://doi.org/10.1182/blood.V81.7.1960.1960.

20. Lord BI, Woolford LB, Wood LM, Czaplewski LG, McCourt M, Hunter MG, et al. Mobilization of early hematopoietic progenitor cells with BB-10010: a genetically engineered variant of human macrophage inflammatory protein-1 alpha. Blood. 1995;85(12):3412-5. https://doi.org/10.1182/blood. V85.12.3412.bloodjournal85123412.

21. Mauch P, Lamont C, Neben TY, Quinto C, Goldman SJ, Witsell A. Hematopoietic stem cells in the blood after stem cell factor and interleukin11 administration: evidence for different mechanisms of mobilization. Blood. 1995;86(12):4674-80. https://doi.org/10.1182/blood.V86.12.4674.bloodjourna 186124674. 
22. Brasel K, McKenna HJ, Charrier K, Morrissey PJ, Williams DE, Lyman SD. Flt3 ligand synergizes with granulocyte-macrophage colony-stimulating factor or granulocyte colony-stimulating factor to mobilize hematopoietic progenitor cells into the peripheral blood of mice. Blood. 1997;90(9):3781-8. https://doi. org/10.1182/blood.V90.9.3781.

23. Sudo Y, Shimazaki C, Ashihara E, Kikuta T, Hirai H, Sumikuma T, et al. Synergistic effect of FLT-3 ligand on the granulocyte colony-stimulating factor-induced mobilization of hematopoietic stem cells and progenitor cells into blood in mice. Blood. 1997;89(9):3186-91. https://doi.org/10.1182/ blood.V89.9.3186.

24. Wang J, Mukaida N, Zhang Y, Ito T, Nakao S, Matsushima K. Enhanced mobilization of hematopoietic progenitor cells by mouse MIP-2 and granulocyte colony-stimulating factor in mice. J Leukoc Biol. 1997;62(4):5039. https://doi.org/10.1002/jlb.62.4.503.

25. Neipp M, Zorina T, Domenick MA, Exner BG, Ildstad ST. Effect of FLT3 ligand and granulocyte colony-stimulating factor on expansion and mobilization of facilitating cells and hematopoietic stem cells in mice: kinetics and repopulating potential. Blood. 1998;92(9):3177-88. https://doi.org/10.1182/ blood.V92.9.3177.

26. Patchen ML, Liang J, Vaudrain T, Martin T, Melican D, Zhong S, et al. Mobilization of peripheral blood progenitor cells by Betafectin PGG-Glucan alone and in combination with granulocyte colony-stimulating factor. Stem Cells. 1998;16(3):208-17. https://doi.org/10.1002/stem.160208.

27. Torii Y, Nitta Y, Akahori H, Tawara T, Kuwaki T, Ogami K, et al. Mobilization of primitive haemopoietic progenitor cells and stem cells with long-term repopulating ability into peripheral blood in mice by pegylated recombinant human megakaryocyte growth and development factor. $\mathrm{Br} J$ Haematol. 1998;103(4):1172-80. https://doi.org/10.1046/j.1365-2141.1998. 01113.x

28. Zhang Y, Zhu J, Zhang Y. Effect of intravenous injection of interleukin-8 on mouse hematopoietic progenitor cell mobilization. Zhonghua Xue Ye Xue Za Zhi. 1998;19(12):642-5.

29. Verma UN, van den Blink B, Pillai R, Chawla J, Mazumder A, Herscowitz HB, et al. Paclitaxel vs cyclophosphamide in peripheral blood stem cell mobilization: comparative studies in a murine model. Exp Hematol. 1999; 27(3):553-60.

30. de Haan G, Ausema A, Wilkens M, Molineux G, Dontje B. Efficient mobilization of haematopoietic progenitors after a single injection of pegylated recombinant human granulocyte colony-stimulating factor in mouse strains with distinct marrow-cell pool sizes. Br J Haematol. 2000; 110(3):638-46. https://doi.org/10.1046/j.1365-2141.2000.02252.x.

31. Frenette $P S$, Weiss L. Sulfated glycans induce rapid hematopoietic progenitor cell mobilization: evidence for selectin-dependent and independent mechanisms. Blood. 2000;96(7):2460-8. https://doi.org/10.1182/ blood.V96.7.2460.

32. Kikuta T, Shimazaki C, Ashihara E, Sudo Y, Hirai H, Sumikuma T, et al. Mobilization of hematopoietic primitive and committed progenitor cells into blood in mice by anti-vascular adhesion molecule-1 antibody alone or in combination with granulocyte colony-stimulating factor. Exp Hematol. 2000;28(3):311-7.

33. Robinson S, Mosley RL, Parajuli P, Pisarev V, Sublet J, Ulrich A, et al. Comparison of the hematopoietic activity of flt-3 ligand and granulocytemacrophage colony-stimulating factor acting alone or in combination. J Hematother Stem Cell Res. 2000;9(5):711-20. https://doi.org/10.1089/152581 60050196759.

34. Sweeney EA, Priestley GV, Nakamoto B, Collins RG, Beaudet AL, Papayannopoulou T. Mobilization of stem/progenitor cells by sulfated polysaccharides does not require selectin presence. Proc Natl Acad Sci U S A. 2000;97(12):6544-9. https://doi.org/10.1073/pnas.97.12.6544.

35. Fleming WH, Mulcahy JM, McKearn JP, Streeter PR. Progenipoietin-1: a multifunctional agonist of the granulocyte colony-stimulating factor receptor and fetal liver tyrosine kinase-3 is a potent mobilizer of hematopoietic stem cells. Exp Hematol. 2001;29(8):943-51.

36. Honda K, Takenaka K, Shinagawa K, Ishimaru F, Ikeda K, Niiya K, et al. Synergistic effects of pegylated recombinant human megakaryocyte growth and development factor and granulocyte colony-stimulating factor on mobilization of hematopoietic progenitor and stem cells with long-term repopulating ability into peripheral blood in mice. Bone Marrow Transplant. 2001;28(4):329-34. https://doi.org/10.1038/sj.bmt.1703140.

37. King AG, Horowitz D, Dillon SB, Levin R, Farese AM, MacVittie TJ, et al. Rapid mobilization of murine hematopoietic stem cells with enhanced engraftment properties and evaluation of hematopoietic progenitor cell mobilization in rhesus monkeys by a single injection of SB-251353, a specific truncated form of the human CXC chemokine GRObeta. Blood. 2001;97(6):1534-42.

38. Carlo-Stella C, Di Nicola M, Magni M, Longoni P, Milanesi M, Stucchi C, et al. Defibrotide in combination with granulocyte colony-stimulating factor significantly enhances the mobilization of primitive and committed peripheral blood progenitor cells in mice. Cancer Res. 2002;62(21):6152-7.

39. Sweeney EA, Lortat-Jacob H, Priestley GV, Nakamoto B, Papayannopoulou T. Sulfated polysaccharides increase plasma levels of SDF-1 in monkeys and mice: involvement in mobilization of stem/progenitor cells. Blood. 2002; 99(1):44-51. https://doi.org/10.1182/blood.V99.1.44.

40. Velders GA, Pruijt JF, Verzaal P, van Os R, van Kooyk Y, Figdor CG, et al. Enhancement of G-CSF-induced stem cell mobilization by antibodies against the beta 2 integrins LFA-1 and Mac-1. Blood. 2002;100(1):327-33.

41. Hofer M, Weiterová L, Vacek A, Znojil V, Pospísil M, Vácha J. Elevation of extracellular adenosine mobilizes haematopoietic progenitor cells and granulocytes into peripheral blood and enhances the mobilizing effects of granulocyte colony-stimulating factor. Eur J Haematol. 2003;71(3):204-10. https://doi.org/10.1034/j.1600-0609.2003.00120.x.

42. Meng FY, Jiang ZJ, Yi ZS, Xu D. Study of murine hematopoietic stem/ progenitor cell mobilized by recombinant human interleukin 11 combination with granulocyte-colony stimulating factor. Zhonghua Xue Ye Xue Za Zhi. 2003;24(5):225-7.

43. Ojeifo JO, Wu AG, Herscowitz HB, Meehan KR. Phenotypic and immunologic characteristics of docetaxel-mobilized peripheral blood stem cells in mice. J Hematother Stem Cell Res. 2003;12(2):189-97. https://doi.org/10.1089/152 581603321628331.

44. Robinson SN, Chavez JM, Pisarev VM, Mosley RL, Rosenthal GJ, Blonder JM, et al. Delivery of Flt3 ligand (Flt3L) using a poloxamer-based formulation increases biological activity in mice. Bone Marrow Transplant. 2003;31(5): 361-9. https://doi.org/10.1038/sj.bmt.1703816.

45. Liu CF, Du ZM, Zhagn YK. Effect of anti-CD49d monoclonal antibody on mobilization of CD34 positive cell into peripheral blood in mice. Zhongguo shi yan xue ye xue za zhi. 2004;12(1):59-62.

46. Nakamura Y, Tajima F, Ishiga K, Yamazaki H, Oshimura M, Shiota G, et al. Soluble c-kit receptor mobilizes hematopoietic stem cells to peripheral blood in mice. Exp Hematol. 2004;32(4):390-6. https://doi.org/10.1016/j. exphem.2004.01.004.

47. Pelus LM, Bian H, King AG, Fukuda S. Neutrophil-derived MMP-9 mediates synergistic mobilization of hematopoietic stem and progenitor cells by the combination of G-CSF and the chemokines GRObeta/CXCL2 and GRObetaT/ CXCL2delta4. Blood. 2004;103(1):110-9. https://doi.org/10.1182/blood-200304-1115.

48. Broxmeyer HE, Orschell CM, Clapp DW, Hangoc G, Cooper S, Plett PA, et al. Rapid mobilization of murine and human hematopoietic stem and progenitor cells with AMD3100, a CXCR4 antagonist. J Exp Med. 2005;201(8): 1307-18. https://doi.org/10.1084/jem.20041385.

49. Pelus LM, Bian H, Fukuda S, Wong D, Merzouk A, Salari H. The CXCR4 agonist peptide, CTCE-0021, rapidly mobilizes polymorphonuclear neutrophils and hematopoietic progenitor cells into peripheral blood and synergizes with granulocyte colony-stimulating factor. Exp Hematol. 2005; 33(3):295-307. https://doi.org/10.1016/j.exphem.2004.11.008.

50. Selleri C, Montuori N, Ricci P, Visconte V, Baiano A, Carriero MV, et al. In vivo activity of the cleaved form of soluble urokinase receptor: a new hematopoietic stem/progenitor cell mobilizer. Cancer Res. 2006;66(22): 10885-90. https://doi.org/10.1158/0008-5472.CAN-06-1311.

51. Abraham M, Biyder K, Begin M, Wald H, Weiss ID, Galun E, et al. Enhanced unique pattern of hematopoietic cell mobilization induced by the CXCR4 antagonist 4F-benzoyl-TN14003. Stem Cells. 2007;25(9):2158-66. https://doi. org/10.1634/stemcells.2007-0161.

52. Broxmeyer HE, Hangoc G, Cooper S, Campbell T, Ito S, Mantel C. AMD3100 and CD26 modulate mobilization, engraftment, and survival of hematopoietic stem and progenitor cells mediated by the SDF-1/CXCL12CXCR4 axis. Ann New York Acad Sci. 2007;1106(1):1-19. https://doi.org/10.11 96/annals.1392.013.

53. Carlo-Stella C, Di Nicola M, Longoni P, Cleris L, Lavazza C, Milani R, et al. Placental growth factor-1 potentiates hematopoietic progenitor cell mobilization induced by granulocyte colony-stimulating factor in mice and nonhuman primates. Stem Cells. 2007;25(1):252-61. https://doi.org/10.1634/ stemcells.2006-0020 
54. Fukuda S, Bian H, King AG, Pelus LM. The chemokine GROß mobilizes early hematopoietic stem cells characterized by enhanced homing and engraftment. Blood. 2007;110(3):860-9. https://doi.org/10.1182/blood-200606-031401.

55. Herbert KE, Walkley CR, Winkler IG, Hendy J, Olsen GH, Yuan YD, et al. Granulocyte colony-stimulating factor and an RARalpha specific agonist, VTP195183, synergize to enhance the mobilization of hematopoietic progenitor cells. Transplantation. 2007;83(4):375-84. https://doi.org/10.1097/ 01.tp.0000251376.75347.b4

56. Kubonishi S, Kikuchi T, Yamaguchi S, Tamamura H, Fujii N, Watanabe T, et al. Rapid hematopoietic progenitor mobilization by sulfated colominic acid. Biochem Biophys Res Commun. 2007;355(4):970-5. https://doi.org/10.1016/j. bbrc.2007.02.069.

57. Brunner S, Zaruba MM, Huber B, David R, Vallaster M, Assmann G, et al. Parathyroid hormone effectively induces mobilization of progenitor cells without depletion of bone marrow. Exp Hematol. 2008;36(9):1157-66. https://doi.org/10.1016/j.exphem.2008.03.014.

58. Cramer DE, Wagner S, Li B, Liu J, Hansen R, Reca R, et al. Mobilization of hematopoietic progenitor cells by yeast-derived beta-glucan requires activation of matrix metalloproteinase-9. Stem Cells. 2008;26(5):1231-40. https://doi.org/10.1634/stemcells.2007-0712.

59. Tjwa M, Janssens $S$, Carmeliet P. Plasmin therapy enhances mobilization of HPCs after G-CSF. Blood. 2008;112(10):4048-50. https://doi.org/10.1182/ blood-2008-07-166587.

60. Albanese P, Caruelle D, Frescaline G, Delbé J, Petit-Cocault L, Huet E, et al. Glycosaminoglycan mimetics-induced mobilization of hematopoietic progenitors and stem cells into mouse peripheral blood: structure/function insights. Exp Hematol. 2009;37(9):1072-83. https://doi.org/10.1016/j. exphem.2009.06.005

61. Bonig H, Chudziak D, Priestley G, Papayannopoulou T. Insights into the biology of mobilized hematopoietic stem/progenitor cells through innovative treatment schedules of the CXCR4 antagonist AMD3100. Exp Hematol. 2009;37(3):402-15.e1.

62. Dygai AM, Vereshchagin El, Zyuz'kov GN, Zhdanov W, Madonov PG, Simanina EV, et al. Mobilization of progenitor cells into the blood by immobilized granulocytic colony-stimulating factor. Bull Exp Biol Med. 2009; 147(4):499-502. https://doi.org/10.1007/s10517-009-0550-y.

63. Huang YH, Liu YC, Yen CF, Chen HC, Chen SJ, R. King CH, et al. Rapid mobilization of murine hematopoietic stem and progenitor cells with TG0054, a novel CXCR4 antagonist. Blood. 2009;114(22):3542.

64. Ramirez P, Rettig MP, Uy GL, Deych E, Holt MS, Ritchey JK, et al. BIO5192, a smal molecule inhibitor of VLA-4, mobilizes hematopoietic stem and progenitor cells. Blood. 2009;114(7):1340-3. https://doi.org/10.1182/blood-2008-10-184721.

65. Yatuv R, Carmel-Goren L, Dayan I, Robinson M, Baru M. Binding of proteins to PEGylated liposomes and improvement of G-CSF efficacy in mobilization of hematopoietic stem cells. J Controlled Release. 2009;135(1):44-50. https:// doi.org/10.1016/j.jconrel.2008.12.004.

66. de Kruijf EJ, Hagoort H, Velders GA, Fibbe WE, van Pel M. Hematopoietic stem and progenitor cells are differentially mobilized depending on the duration of Flt3-ligand administration. Haematologica. 2010;95(7):1061-7. https://doi.org/10.3324/haematol.2009.016691.

67. Ryan MA, Nattamai KJ, Xing E, Schleimer D, Daria D, Sengupta A, et al. Pharmacological inhibition of EGFR signaling enhances G-CSF-induced hematopoietic stem cell mobilization. Nat Med. 2010;16(10):1141-6. https:// doi.org/10.1038/nm.2217.

68. Tchernychev B, Ren Y, Sachdev P, Janz JM, Haggis L, O'Shea A, et al. Discovery of a CXCR4 agonist pepducin that mobilizes bone marrow hematopoietic cells. Proc Natl Acad Sci U S A. 2010;107(51):22255-9. https:// doi.org/10.1073/pnas.1009633108

69. Berchanski A, Kalinkovich A, Ludin A, Lapidot T, Lapidot A. Insights into the mechanism of enhanced mobilization of hematopoietic progenitor cells and release of CXCL12 by a combination of AMD3100 and aminoglycosidepolyarginine conjugates. FEBS J. 2011;278(21):4150-65. https://doi.org/1 0.1111/j.1742-4658.2011.08348.x.

70. Chigaev A, Wu Y, Williams DB, Smagley Y, Sklar LA. Discovery of very late antigen-4 (VLA-4, alpha4beta1 integrin) allosteric antagonists. J Biol Chem. 2011;286(7):5455-63. https://doi.org/10.1074/jbc.M110.162636.

71. Dar A, Schajnovitz A, Lapid K, Kalinkovich A, Itkin T, Ludin A, et al. Rapid mobilization of hematopoietic progenitors by AMD3100 and catecholamines is mediated by CXCR4-dependent SDF-1 release from bone marrow stromal cells. Leukemia. 2011;25(8):1286-96. https://doi.org/10.1038/leu.2011.62.
72. Di Giacomo F, Lewandowski D, Cabannes E, Nancy-Portebois V, Petitou M, Fichelson S, et al. Heparan sulfate mimetics can efficiently mobilize longterm hematopoietic stem cells. Haematologica. 2012;97(4):491-9.

73. Juarez JG, Harun N, Thien M, Welschinger R, Baraz R, Pena AD, et al. Sphingosine-1-phosphate facilitates trafficking of hematopoietic stem cells and their mobilization by CXCR4 antagonists in mice. Blood. 2012;119(3): 707-16. https://doi.org/10.1182/blood-2011-04-348904.

74. Lucas D, Bruns I, Battista M, Mendez-Ferrer S, Magnon C, Kunisaki Y, et al. Norepinephrine reuptake inhibition promotes mobilization in mice: potential impact to rescue low stem cell yields. Blood. 2012;119(17):3962-5. https://doi.org/10.1182/blood-2011-07-367102.

75. Chen C, Cao J, Song X, Zeng L, Li Z, Li Y, et al. Adrenaline administration promotes the efficiency of granulocyte colony stimulating factor-mediated hematopoietic stem and progenitor cell mobilization in mice. Int J Hematol. 2013:97(1):50-7. https://doi.org/10.1007/s12185-012-1228-1.

76. Dygai AM, Zyuz'kov GN, Zhdanov W, Udut EV, Miroshnichenko LA, Simanina EV, et al. Specific activity of electron-beam synthesis immobilized hyaluronidase on G-CSF induced mobilization of bone marrow progenitor cells. Stem Cell Rev Rep. 2013;9(2):140-7.

77. Hoggatt J, Mohammad KS, Singh P, Hoggatt AF, Chitteti BR, Speth JM, et al. Differential stem- and progenitor-cell trafficking by prostaglandin E2. Nature. 2013;495(7441):365-9.

78. Karpova D, Dauber K, Spohn G, Chudziak D, Wiercinska E, Schulz M, et al. The novel CXCR4 antagonist POL5551 mobilizes hematopoietic stem and progenitor cells with greater efficiency than Plerixafor. Leukemia. 2013; 27(12):2322-31. https://doi.org/10.1038/leu.2013.266.

79. Kook S, Cho J, Lee SB, Lee BC. The nucleotide sugar UDP-glucose mobilizes long-term repopulating primitive hematopoietic cells. J Clin Investig. 2013; 123(8):3420-35. https://doi.org/10.1172/JCl64060.

80. Vater A, Sahlmann J, Kröger N, Zöllner S, Lioznov M, Maasch C, et al. Hematopoietic stem and progenitor cell mobilization in mice and humans by a first-in-class mirror-image oligonucleotide inhibitor of CXCL12. Clin Pharmacol Ther. 2013;94(1):150-7. https://doi.org/10.1038/clpt.2013.58.

81. Chen C, Song X, Ma S, Wang X, Xu J, Zhang H, et al. Cdc42 inhibitor ML141 enhances G-CSF-induced hematopoietic stem and progenitor cell mobilization. Int J Hematol. 2014;101(1):5-12. https://doi.org/10.1007/s121 85-014-1690-z.

82. Ghobadi A, Rettig MP, Cooper ML, Holt MS, Ritchey JK, Eissenberg L, et al. Bortezomib is a rapid mobilizer of hematopoietic stem cells in mice via modulation of the VCAM-1/NLA-4 axis. Blood. 2014;124(17):2752-4. https:// doi.org/10.1182/blood-2014-08-595967.

83. He S, Chu J, Vasu S, Deng Y, Yuan S, Zhang J, et al. FLT3L and plerixafor combination increases hematopoietic stem cell mobilization and leads to improved transplantation outcome. Biol Blood Marrow Transplant. 2014; 20(3):309-13. https://doi.org/10.1016/j.bbmt.2013.11.024.

84. Saez B, Ferraro F, Yusuf RZ, Cook CM, Yu WW, Pardo-Saganta A, et al. Inhibiting stromal cell heparan sulfate synthesis improves stem cell mobilization and enables engraftment without cytotoxic conditioning. Blood. 2014;124(19):2937-47. https://doi.org/10.1182/blood-2014-08-593426.

85. Zhang J, Ren X, Shi W, Wang S, Chen H, Zhang B, et al. Small molecule Me6TREN mobilizes hematopoietic stem/progenitor cells by activating MMP-9 expression and disrupting SDF-1/CXCR4 axis. Blood. 2014;123(3): 428-41. https://doi.org/10.1182/blood-2013-04-498535.

86. Forristal CE, Nowlan B, Jacobsen RN, Barbier V, Walkinshaw G, Walkley CR, et al. HIF-1a is required for hematopoietic stem cell mobilization and 4prolyl hydroxylase inhibitors enhance mobilization by stabilizing HIF-1a. Leukemia. 2015;29(6):1366-78. https://doi.org/10.1038/leu.2015.8.

87. Santiago-Osorio E, Ledesma-Martínez E, Aguiñiga-Sánchez I, Poblano-Pérez I, Weiss-Steider B, Montesinos-Montesinos JJ, et al. Sodium caseinate (CasNa) induces mobilization of hematopoietic stem cells in a BALB/c mouse model. Med Sci Monit Basic Res. 2015;21:206-12. https://doi.org/10.12659/MSMBR. 895442

88. Wysoczynski M, Ratajczak J, Pedziwiatr D, Rokosh G, Bolli R, Ratajczak MZ. Identification of heme oxygenase $1(\mathrm{HO}-1)$ as a novel negative regulator of mobilization of hematopoietic stem/progenitor cells. Stem Cell Rev Rep. 2015;11(1):110-8.

89. Xing S, Xiong GL, Shen X, Liu XL, Yang M, Dong B, et al. Mobilization effect of HS6101 on hematopoietic cells of mice. Zhongguo shi yan xue ye xue za zhi. 2015;23(5):1427-31. https://doi.org/10.7534/j.issn.1009-2137.2015.05.039.

90. Cao B, Zhang Z, Grassinger J, Williams B, Heazlewood CK, Churches QI, et al. Therapeutic targeting and rapid mobilization of endosteal HSC using a 
small molecule integrin antagonist. Nature Commun. 2016;7(1):11007. https://doi.org/10.1038/ncomms11007.

91. Lu XJ, Chen Q, Rong YJ, Yang GJ, Li CH, Xu NY, et al. LECT2 drives haematopoietic stem cell expansion and mobilization via regulating the macrophages and osteolineage cells. Nature commun. 2016;7:12719.

92. Yan BZ, Ma HM, Kong CQ, Liang Y, Zhu WY, Jiang ST. Combination effect of AMD3100 and dexamethasone on the mobilization of hematopoietic stem cells. Chinese J Tissue Eng Res. 2016;20(36):5351-7.

93. Adamiak M, Chelvarajan L, Lynch KR, Santos WL, Abdel-Latif A, Ratajczak MZ. Mobilization studies in mice deficient in sphingosine kinase 2 support a crucial role of the plasma level of sphingosine-1-phosphate in the egress of hematopoietic stem progenitor cells. Oncotarget. 2017;8(39):65588-600. https://doi.org/10.18632/oncotarget.19514

94. Karpova D, Ritchey JK, Holt MS, Abou-Ezzi G, Monlish D, Batoon L, et al. Continuous blockade of CXCR4 results in dramatic mobilization and expansion of hematopoietic stem and progenitor cells. Blood. 2017;129(21): 2939-49. https://doi.org/10.1182/blood-2016-10-746909.

95. Nowlan B, Futrega K, Brunck ME, Walkinshaw G, Flippin LE, Doran MR, et al. HIF-1a-stabilizing agent FG-4497 rescues human CD34(+) cell mobilization in response to G-CSF in immunodeficient mice. Exp Hematol. 2017;52:50-5. e6.

96. Ogle ME, Olingy CE, Awojoodu AO, Das A, Ortiz RA, Cheung HY, et al. Sphingosine-1-phosphate receptor-3 supports hematopoietic stem and progenitor cell residence within the bone marrow niche. Stem Cells. 2017; 35(4):1040-52. https://doi.org/10.1002/stem.2556.

97. Redpath AN, François M, Wong SP, Bonnet D, Rankin SM. Two distinct CXCR4 antagonists mobilize progenitor cells in mice by different mechanisms. Blood Adv. 2017;1 (22):1934-43.

98. Wang W, Yu S, Myers J, Wang Y, Xin WW, Albakri M, et al. Notch2 blockade enhances hematopoietic stem cell mobilization and homing. Haematologica. 2017;102(10):1785-95. https://doi.org/10.3324/haematol.201 7.168674 .

99. Adamiak M, Bujko K, Cymer M, Plonka M, Glaser T, Kucia M, et al. Novel evidence that extracellular nucleotides and purinergic signaling induce innate immunity-mediated mobilization of hematopoietic stem/progenitor cells. Leukemia. 2018;32(9):1920-31. https://doi.org/10.1038/s41375-018-0122-0.

100. Ghobadi A, Rettig MP, Holt MS, Ritchey JK, Kennerly K, Chendamarai E, et al. Ixazomib, an oral proteasome inhibitor, induces rapid mobilization of hematopoietic progenitor cells in mice. Blood. 2018;131(23):2594-6.

101. Hoggatt J, Singh P, Tate TA, Chou BK, Datari SR, Fukuda S, et al. Rapid mobilization reveals a highly engraftable hematopoietic stem cell. Cell. 2018;172(1-2):191-204.e10.

102. Notario L, Alari-Pahissa E, Albentosa A, Leiva M, Sabio G, Lauzurica P. AntiCD69 therapy induces rapid mobilization and high proliferation of HSPCS through S1P and mTOR. Leukemia. 2018:32(6):1445-57.

103. Wu CH, Song JS, Kuan HH, Wu SH, Chou MC, Jan JJ, et al. Development of stem-cell-mobilizing agents targeting CXCR4 receptor for peripheral blood stem sell transplantation and beyond. J Med Chem. 2018;61(3):818-33.

104. Adamiak M, Bujko K, Brzezniakiewicz-Janus K, Kucia M, Ratajczak J, Ratajczak MZ. The inhibition of CD39 and CD73 cell surface ectonucleotidases by small molecular inhibitors enhances the mobilization of bone marrow residing stem cells by decreasing the extracellular level of adenosine. Stem Cell Rev Rep. 2019:15(6):892-9.

105. Alt C, Yuan S, Wu F, Stankovich B, Calaoagan J, Schopies S, et al. Longacting IL-33 mobilizes high-quality hematopoietic stem and progenitor cells more efficiently than granulocyte colony-stimulating factor or AMD3100. Biol Blood Marrow Transplant. 2019:25(8):1475-85.

106. Bisht K, Brunck ME, Matsumoto T, McGirr C, Nowlan B, Fleming W, et al. HIF prolyl hydroxylase inhibitor FG-4497 enhances mouse hematopoietic stem cell mobilization via VEGFR2/KDR. Blood Adv. 2019:3(3):406-18.

107. Karpova D, Rettig MP, Ritchey J, Cancilla D, Christ S, Gehrs L, et al. Targeting VLA4 integrin and CXCR2 mobilizes serially repopulating hematopoietic stem cells. J Clin Investig. 2019;129(7):2745-59. https://doi.org/10.1172/ JCl124738.

108. Liu W, Du W. Rational identification of a Cdc42 inhibitor presents a new regimen for long-term hematopoietic stem cell mobilization. Diabetes. 2019;33(3):749-61.

109. Müller TA, Pennisi S, Zwick A, Decker S, Klein C, Rister B, et al. PIM1 inhibition effectively enhances plerixafor-induced HSC mobilization by counteracting CXCR4 upregulation and blocking CXCL12 secretion. Leukemia. 2019;33(5):1296-301.
110. Smith-Berdan S, Bercasio A, Rajendiran S, Forsberg EC. Viagra enables efficient, single-day hematopoietic stem cell mobilization. Stem cell Rep. 2019;13(5):787-92.

111. Szade A, Szade K, Nowak WN, Bukowska-Strakova K, Muchova L, Gońka M, et al. Cobalt protoporphyrin IX increases endogenous G-CSF and mobilizes HSC and granulocytes to the blood. EMBO Mol Med. 2019;11(12):e09571.

112. Fang X, Fang X, Mao Y, Ciechanover A, Xu Y, An J, et al. A novel small molecule CXCR4 antagonist potently mobilizes hematopoietic stem cells in mice and monkeys. Stem Cell Res Ther. 2021;12(1):17.

113. Kaur S, Sehgal A, Wu AC, Millard SM, Batoon L, Sandrock CJ, et al. Stable colony-stimulating factor 1 fusion protein treatment increases hematopoietic stem cell pool and enhances their mobilisation in mice. J Hematol Oncol. 2021;14(1):3.

114. Pesek G, Cottler-Fox M. Hematopoietic stem cell mobilization: a clinical protocol. Methods Mol Biol. 2012;904:69-77. https://doi.org/10.1007/978-161779-943-3_5.

115. Forristal CE, Levesque JP. Targeting the hypoxia-sensing pathway in clinical hematology. Stem Cells Transl Med. 2014;3(2):135-40. https://doi.org/10. 5966/sctm.2013-0134.

116. Bilgin YM, de Greef GE. Plerixafor for stem cell mobilization: the current status. Curr Opin Hematol. 2016;23(1):67-71. https://doi.org/10.1097/MOH. 0000000000000200

117. Donahue RE, Jin P, Bonifacino AC, Metzger ME, Ren J, Wang E, et al. Plerixafor (AMD3100) and granulocyte colony-stimulating factor (G-CSF) mobilize different CD34+ cell populations based on global gene and microRNA expression signatures. Blood. 2009;114(12):2530-41. https://doi. org/10.1182/blood-2009-04-214403.

118. Kean LS, Sen S, Onabajo O, Singh K, Robertson J, Stempora L, et al. Significant mobilization of both conventional and regulatory $T$ cells with AMD3100. Blood. 2011;118(25):6580-90. https://doi.org/10.1182/blood-201106-359331.

119. DiPersio JF, Micallef IN, Stiff PJ, Bolwell BJ, Maziarz RT, Jacobsen E, et al. Phase III prospective randomized double-blind placebo-controlled trial of plerixafor plus granulocyte colony-stimulating factor compared with placebo plus granulocyte colony-stimulating factor for autologous stem-cell mobilization and transplantation for patients with non-Hodgkin's lymphoma. J Clin Oncol. 2009;27(28):4767-73. https://doi.org/10.1200/JCO.2 008.20.7209.

120. DiPersio JF, Stadtmauer EA, Nademanee A, Micallef IN, Stiff PJ, Kaufman JL, et al. Plerixafor and G-CSF versus placebo and G-CSF to mobilize hematopoietic stem cells for autologous stem cell transplantation in patients with multiple myeloma. Blood. 2009;113(23):5720-6. https://doi. org/10.1182/blood-2008-08-174946.

121. Zhu J, Huang $H$, Chen $H$, Zhang $X$, Li Z, Wu D, et al. Plerixafor and granulocyte-colony-stimulating factor for mobilization of hematopoietic stem cells for autologous transplantation in Chinese patients with nonHodgkin's lymphoma: a randomized Phase 3 study. Transfusion. 2018;58(1): 81-7. https://doi.org/10.1111/trf.14426.

122. Giralt S, Costa L, Schriber J, Dipersio J, Maziarz R, McCarty J, et al. Optimizing autologous stem cell mobilization strategies to improve patient outcomes: consensus guidelines and recommendations. Biol Blood Marrow Transplant. 2014;20(3):295-308. https://doi.org/10.1016/j.bbmt.2013.10.013.

123. Duarte RF, Shaw BE, Marín P, Kottaridis P, Ortiz M, Morante C, et al. Plerixafor plus granulocyte CSF can mobilize hematopoietic stem cells from multiple myeloma and lymphoma patients failing previous mobilization attempts: EU compassionate use data. Bone Marrow Transplant. 2011;46(1):52-8. https:// doi.org/10.1038/bmt.2010.54

\section{Publisher's Note}

Springer Nature remains neutral with regard to jurisdictional claims in published maps and institutional affiliations. 